\title{
Geochronology and Geochemistry of the Zengudi and Tuobake Granite Porphyries in the Sanjiang Region, SW China: Petrogenesis and Tectonic Significance
}

\author{
Mao-Jun Tian ${ }^{1}$, Huan Li ${ }^{1, *(D)}$, Landry Soh Tamehe ${ }^{1(D)}$ and Zhen $\mathrm{Xi}^{2}$ (D) \\ 1 Key Laboratory of Metallogenic Prediction of Nonferrous Metals and Geological Environment Monitoring, \\ Ministry of Education, School of Geosciences and Info-Physics, Central South University, Changsha 410083, \\ China; tianmj@csu.edu.cn (M.-J.T.); tamehelandry@yahoo.com (L.S.T.) \\ 2 School of Municipal and Geomatics Engineering, Hunan City University, Yiyang 413000, China; \\ xizhen@hncu.edu.cn \\ * Correspondence: lihuan@csu.edu.cn
}

\section{check for} updates

Citation: Tian, M.-J.; Li, H.; Tamehe, L.S.; Xi, Z. Geochronology and Geochemistry of the Zengudi and Tuobake Granite Porphyries in the Sanjiang Region, SW China:

Petrogenesis and Tectonic Significance. Minerals 2021, 11, 404. https://doi.org/10.3390/ $\min 11040404$

Academic Editor: Dominique Gasquet

Received: 26 February 2021

Accepted: 9 April 2021

Published: 12 April 2021

Publisher's Note: MDPI stays neutral with regard to jurisdictional claims in published maps and institutional affiliations.

Copyright: (c) 2021 by the authors. Licensee MDPI, Basel, Switzerland. This article is an open access article distributed under the terms and conditions of the Creative Commons Attribution (CC BY) license (https:// creativecommons.org/licenses/by/ $4.0 /)$.

\begin{abstract}
The boundary between the Gondwana and Yangtze plate is still controversial. In southwest China, the Sanjiang region marks the collision zone which accreted several blocks coming from the northern Gondwana margin. In this region, subduction of the Paleo-Tethys Ocean and associated continental blocks during the Triassic Period led to the formation of an N-S trending complex involving intrusive and volcanic rocks. The intrusive rocks are important for constraining the evolution of the Paleo-Tethyan in southwestern China. This study presents new geochronological, geochemical, and Sr-Nd-Hf isotopic data of granite porphyries from northern Lancangjiang, in order to discuss the origin of these granites and their tectonic significance. Representative samples of the Zengudi and the Tuobake granite porphyries from the Yezhi area yielded weighted mean ${ }^{206} \mathrm{~Pb} /{ }^{238} \mathrm{U}$ ages of 247-254 Ma and $246 \mathrm{Ma}$, respectively. The Zengudi granite porphyries display zircon $\varepsilon_{\mathrm{Hf}}(\mathrm{t})$ values of -12.94 to $-2.63, \varepsilon_{\mathrm{Nd}}(\mathrm{t})$ values of -14.5 to -9.35 , and initial ${ }^{87} \mathrm{Sr} /{ }^{86} \mathrm{Sr}$ ratios of 0.708 to 0.716. The Tuobake granite porphyries have zircon $\varepsilon_{\mathrm{Hf}}(\mathrm{t})$ values of -14.06 to $-6.55, \varepsilon_{\mathrm{Nd}}(\mathrm{t})$ values of -10.9 to -9.41 , and initial ${ }^{87} \mathrm{Sr} /{ }^{86} \mathrm{Sr}$ ratios of 0.716 to 0.731 . Both the Zengudi and Tuobake granite porphyries exhibit strongly peraluminous signatures with high $\mathrm{A} / \mathrm{CNK} n \mathrm{Al}_{2} \mathrm{O}_{3} /\left(\mathrm{K}_{2} \mathrm{O}+\mathrm{Na}_{2} \mathrm{O}+\right.$ $\mathrm{K}_{2} \mathrm{O}$ ) ratios (1.07-1.86 and 0.83-1.33, respectively). These granites are enriched in $\mathrm{Rb}$ and $\mathrm{Th}$, and depleted in $\mathrm{Ti}, \mathrm{Nb}, \mathrm{Ta}, \mathrm{Sr}$, and $\mathrm{P}$, with negative Eu anomalies $\left(\mathrm{Eu} / \mathrm{Eu}^{*}<0.61\right)$. These geochemical and isotopic data indicate that the primary magma of the granite porphyries originated from partial melting of ancient continental crust as a result of basaltic magma underplating and underwent fractionation crystallization during their emplacement. We propose that the Triassic subduction of the Paleo-Tethys Ocean led to crust shortening and thickening in the Sanjiang region, while the northern Lancangjiang area was involved in the continental collision after the subduction of the Paleo-Tethys Ocean before 254 Ma.
\end{abstract}

Keywords: zircon U-Pb dating; granite porphyry; northern Lancangjiang; southern China; tectonic evolution; Paleo-Tethys

\section{Introduction}

The convergent subduction zone is an important window for understanding the interaction between the crust and mantle as well as deciphering the relationship between the magmatic rocks and their tectonic environment [1-3]. In subduction zones, the variability of plate tectonic processes, magma sources, thermal states, and crust thicknesses in the volcanic arc constrained the magmatic activity and led to the segmentation, heterogeneity, and differences of igneous rocks [4-7]. Nowadays, exploring the plate tectonic processes by studying the origin and evolution of igneous rocks in orogenic belts is a hot topic for the tectonic history of continents [8-11]. 
At the Paleo-Tethys stage, the Lhasa, West and East. Qiangtang, Songpan-Ganzi blocks became merged in North Sanjiang, while West. Burma Sibumasu and Indochina blocks became merged in the south of Sanjiang, which related the accreted orogenic process. During the late Cretaceous to Paleogene, continent-continent collision from the India block to south China block led to an orogenic belt collision in the Sanjiang area. Previous research has demonstrated that the magmatism of the Sanjiang area was related to the opening of the Paleo-Tethys Ocean, which occurred between 272 and 207 Ma [12-25]. In this framework, the Paleo-Tethys magmatism mainly occurred from Late Permian to Late Triassic in the Sanjiang region [15-17,19-22]. Magmatic rocks define distinct spatial distribution in the southern and northern segments of this region [21], with relatively late stage of magmatism identified in the southern part compared to the northern portion [17]. Many of these studies were focused on the volcanic rocks of the northern segment of this region $[12,19,20]$, while little research was conducted on granitic rocks. Although the temporal evolution of magma in the arc volcanic belts of the Sanjiang region and the deep dynamic processes have been intensely studied and discussed [6,12-20,22,23], no consensus has been reached on the accurate timing and location of these volcanic arcs related to the Paleo-Tethys. On the other hand, the boundary of the Paleo-Tethys suture zone between the Baoshan and Simao blocks at the Yeshi area is not clear. Thus, a systematic geochemical study of the granitic rocks from specific areas would shed new insight into the tectonic evolution of the Sanjiang region.

The Yezhi area is located at the junction of the Chongshan shear zone and the Jinshajiang fault zone in the northern section of the Jiangda-Weixi volcanic arc belt (Figure 1a,b). Volcanic and granitic rocks generated by variable tectonic processes are exposed in this area, with complex contact relationships due to a strong collision and extrusion during the closure of the Paleo-Tethys Ocean. Exploring the petrogenesis of these rocks is very important to understand the geodynamic setting, subduction, and collision processes of the Yezhi area. In this paper, we present new zircon $\mathrm{U}-\mathrm{Pb}$ age and $\mathrm{Hf}$ isotopic data as well as whole-rock major, trace element, and $\mathrm{Sr}-\mathrm{Nd}$ isotopic data of the Zengudi and Tuobake granite porphyries from the Yezhi area, aiming to: (1) constrain their formation age, (2) characterize their magma source and petrogenesis, and (3) investigate their tectonic setting and provide implications for the Paleo-Tethys evolution.

\section{Geological Setting}

The Sanjiang region in southwest China has been generally considered a convergent margin subduction zone, which was involved during the tectonic evolution process of the Tethys (Figure 1a) [6]. This region can be further regarded as an excellent natural laboratory for studying the subduction-related tectonic processes. In the accretionary orogenic process caused by the collision of archipelagic arc-basin systems during Early Devonian to Middle Triassic [25], the Sanjiang region has experienced ocean-crust subduction and continent-continent collision [16,21], followed by complex thermal magmatism and metamorphism. Previous works on the tectonic history of this region have documented the formation of the Ganzi-Litang suture zone between the south China and Zhongza blocks $[16,26,27]$, the Lancangjiang-Changning-Menglian suture zone between the Baoshan and Simao blocks [28,29], the Jinshajiang [12,30-32] and the Ailaoshan-Songma suture zones between the Simao and Yangtze blocks [33-35] (Figure 1b,d). The LancangjiangChangning-Menglian and the Jinshajiang-Ailaoshan Oceans were opened during the EarlyMiddle Devonian and closed at Middle Triassic. While the Ganzi-Litang and Songma Oceans (southern extension of the Ailaoshan Ocean) were opened during the Early-Middle Devonian and closed at late Triassic. The remnant of the oceanic crust distributed in these suture zones has been systematically studied [6]. Three volcanic arc belts related to the subduction of the Paleo-Tethys Ocean were delineated in the Sanjiang region: the YushuYidun belt formed during Late Triassic (230-200 Ma) [25], the Jiangda-Weixi belt formed during Late Permian to Triassic (270-200 Ma) [21,36], and the Yunxian-Lvchun-Ailaoshan belt formed during Permian to Early-Middle Triassic (285-265 Ma and 260-230 Ma) [20,21]. 
Among these belts, the Jiangda-Weixi belt is the most complex volcanic arc characterized by fragmented blocks [6], which explains that its tectonic evolution is still unclear.
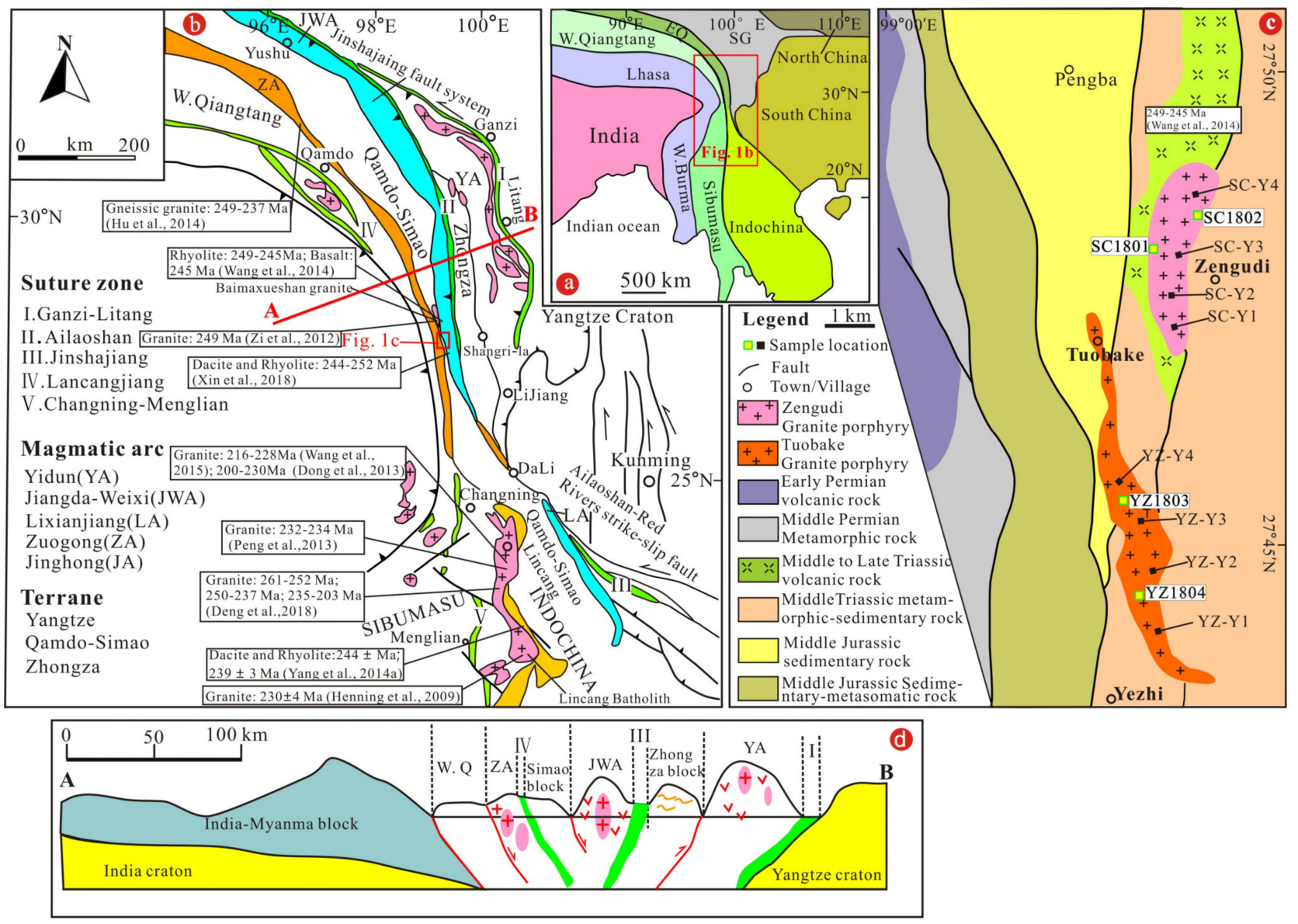

Figure 1. (a) Distribution of main continental blocks and sutures of the East Tethyan belt (modified from [26]); (b) tectonic framework of the Sanjiang region in southwest China showing the major terranes, suture zones, arc volcanic belts, Cenozoic igneous rocks, and location of the Yezhi area (modified from [24]); (c) Simplified geological map showing the Zengudi and Tuobake granite porphyries in the Yezhi area. (a) the east of india myanma block (b) the west of Litang suture zone. (d) An E-W vertical cross-section of the Sanjiang area.

The Zengudi and Tuobake granite porphyries are located in the Yezhi area in the north of the Sanjiang region. The Yezhi area is situated between the Jinshajiang suture zone and the Biluoxueshan-Chongshan shear zone and connects the Zhongzan and Lanping-Simao Blocks on the east and west, respectively (Figure 1a,b). The Yezhi area has undergone intense orogenic events in the East Tethyan tectonic zone as results of oceanic subduction and continental collision from Paleozoic to Mesozoic $[16,19,21]$. These orogenic activities were developed together with a remarkable volume of magmatic movements. Ophiolite and mafic rocks were crystallized in the Late Permian, whereas basic-acidic bimodal volcanic rocks and pyroclastic rocks were formed in the Early-Middle Triassic [16]. A series of ultrabasic, intermediate, and alkaline rocks are also exposed in the area $[16,17]$. Granitic rocks are mostly distributed on the west side of the study area. This region has alternatively experienced multiple periods of magmatism and sedimentation, which led to volcaniclastic successions $[21,34]$. Besides magmatic rocks, the exposed strata encompass Early to Middle Permian metamorphic sandstone, slate, and red sandstone (Figure 1c). The sedimentary succession is characterized by flysch and molasse [25].

The granite porphyries in the Yezhi area are distributed along the east side of the Jiangda-Weixi volcanic arc (Figure 1c). They form a long strip shape from south to north. The Zengudi granite porphyry intrudes into the Triassic Pantiange Formation volcanic rocks 
with an ellipsoid shape and is adjacent to the Triassic Shanglan formation metamorphicsedimentary rocks on the west (Figures $1 \mathrm{c}$ and 2a). The Tuobake granite porphyry displays an irregular long strip shape at the surface, intrusively connected to the Shanglan Formation (Figures $1 c$ and 2b).
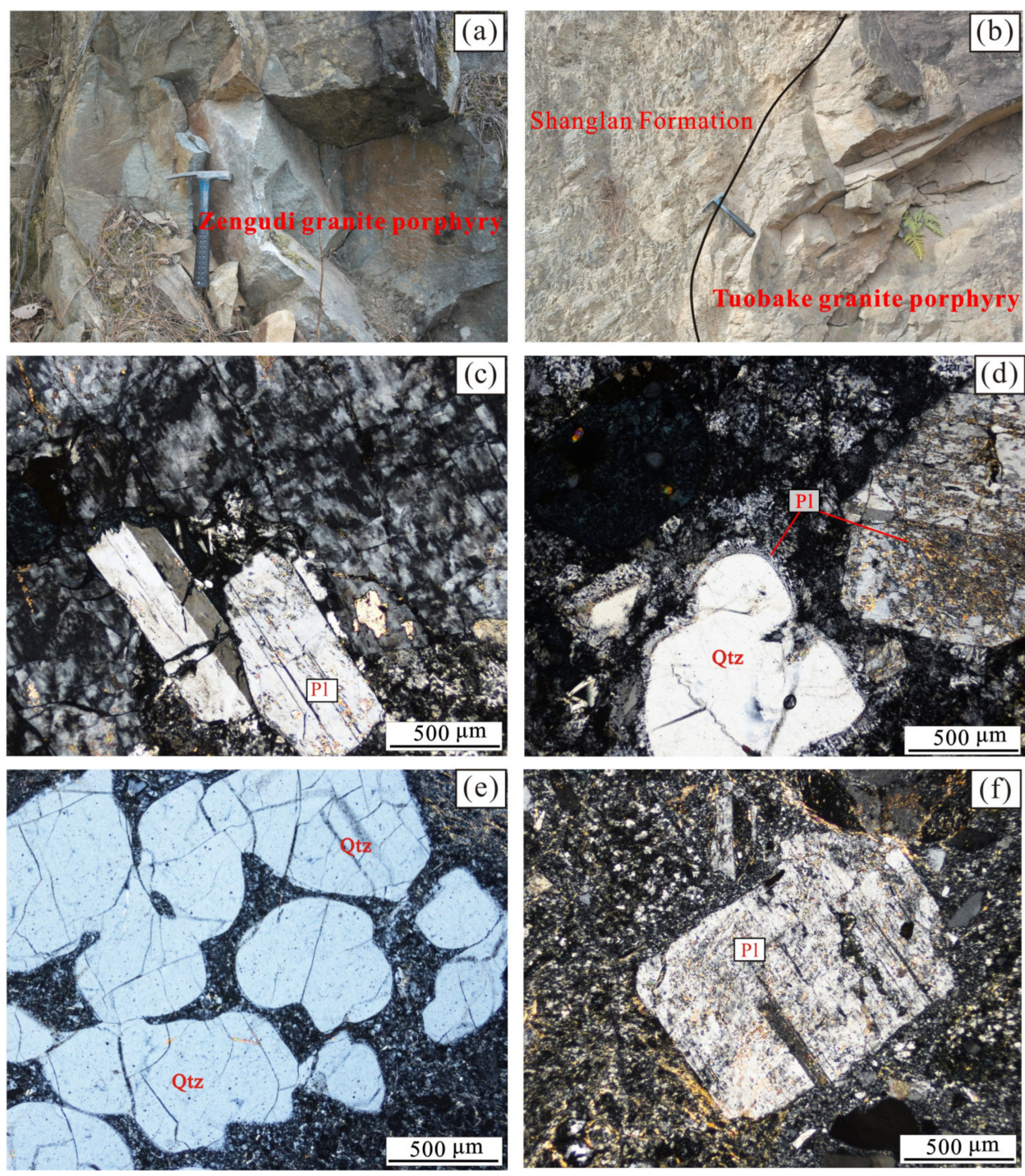

Figure 2. Field occurrences $(\mathbf{a}, \mathbf{b})$ and photomicrographs (c-f) of the Zengudi and Tuobake granite porphyries. (a) The Zengudi granite porphyry shows a massive structure; (b) the Tuobake granite porphyry displays a massive structure and has an intrusive contact with the Shanglan formation; (c) the plagioclase $(\mathrm{Pl})$ is carlsbad-albite twin; (d) the quartz (Qtz) phenocryst is surrounded by plagioclase; (e) the quartz phenocrysts are subcircular due to dissolution; and (f) the plagioclase is a polysynthetic twin.

\section{Samples and Analytical Methods}

\subsection{Sample Description}

The crystals are mainly composed of quartz, plagioclase, K-feldspar, chlorite, and minor opaque minerals (Figure 2c). The quartz is xenomorphic granular and partly clastic with a particle size ranging between 0.3 to $2.0 \mathrm{~mm}$ (Figure 2d). Most plagioclase crystals occur as automorphic or subhedral laths $(0.4-1.5 \mathrm{~mm}$ in size) showing polysynthetic and carlsbad-albite twins, with weakly sericitization and argillization. K-feldspar is mainly characterized by 0.2 to $1.5 \mathrm{~mm}$ automorphic or subhedral carlsbad twinning laths, and 
some crystals are xenomorphic granular. Chlorite has a lamellar shape with a size ranging between 0.2 and $2.0 \mathrm{~mm}$.

The Tuobake granite porphyry samples also have a phanerocrystalline texture with a large amount of felsic minerals (approximately 80\%) and less phenocrysts (approximately $10 \%$ ) of the matrix. The crystals mostly comprise quartz, plagioclase, and K-feldspar. Most quartz crystals $(0.4-2.5 \mathrm{~mm}$ in size) are rounded, resulting from excessive melting corrosion (Figure 2e). Plagioclase often occurs as automorphic or subhedral laths with a particle size ranging between 0.5 and $2.0 \mathrm{~mm}$, displaying polysynthetic and carlsbad-albite twins (Figure 2f). K-feldspar crystals also appear as automorphic or subhedral carlsbad-albite twinning laths (0.4-1.5 $\mathrm{mm}$ in size), and some crystals are granular.

\subsection{Analytical Procedures}

We selected ten rock samples (five Zengudi and five Tuobake granite porphyries) for whole-rock geochemical analyses. Major element concentrations were determined using an X-ray fluorescence (XRF) instrument (Primus II, Rigaku, Japan) at the Wuhan Sample Solution Analytical Technology Co., Ltd. (Wuhan, China). The detailed sample digestion procedure was as follows: (1) the sample powder (200 mesh) was dried in an oven at $105{ }^{\circ} \mathrm{C}$ for $12 \mathrm{~h}$; (2) approximately $1 \mathrm{~g}$ of dried sample was accurately weighed and placed in the ceramic crucible and then heated in a muffle furnace at $1000^{\circ} \mathrm{C}$ for $2 \mathrm{~h}$. After cooling to $400{ }^{\circ} \mathrm{C}$, this sample was placed in the drying vessel and weighed again to calculate the loss on ignition (LOI); (3) $0.6 \mathrm{~g}$ sample powder was mixed with $6 \mathrm{~g}$ co-solvent $\left(\mathrm{Li}_{2} \mathrm{~B}_{4} \mathrm{O}_{7}: \mathrm{LiBO}_{2}: \mathrm{LiF}=9: 2: 1\right)$ and $300 \mathrm{mg}$ oxidant $\left(\mathrm{NH}_{4} \mathrm{NO}_{3}\right)$ in a Pt crucible, which was placed in the furnace at $1150{ }^{\circ} \mathrm{C}$ for $14 \mathrm{~min}$. Then, the air quenching was applied to the sample for $1 \mathrm{~min}$ to produce flat discs on the fire brick for the XRF analyses.

Trace element compositions were also obtained at the Wuhan Sample Solution Analytical Technology Co., Ltd. using an Agilent 7700e ICP-MS instrument. The detailed sample digestion procedure was as follows: (1) the sample powder (200 mesh) was dried in an oven at $105{ }^{\circ} \mathrm{C}$ for $12 \mathrm{~h}$; (2) $0.05 \mathrm{~g}$ sample powder was accurately weighed and placed in a Teflon bomb; (3) $1 \mathrm{~mL} \mathrm{HNO}_{3}$ and $1 \mathrm{~mL} \mathrm{HF}$ were slowly added into the Teflon bomb; (4) the Teflon bomb was put in a stainless steel pressure jacket and heated at $190{ }^{\circ} \mathrm{C}$ in an oven for $>24 \mathrm{~h}$; (5) after cooling, the Teflon bomb was opened and placed on a hot plate at $140{ }^{\circ} \mathrm{C}$ to dry the sample. The $1 \mathrm{~mL} \mathrm{HNO}_{3}$ was added to the sample, which was dried again; (6) $1 \mathrm{~mL}$ $\mathrm{HNO}_{3}, 1 \mathrm{~mL} \mathrm{MQ}$ water, and internal standard solution (1 ppm) were added to the sample and the Teflon bomb was resealed and placed in the oven at $190^{\circ} \mathrm{C}$ for $>12 \mathrm{~h}$; (7) the final solution was transferred to a polyethylene bottle and diluted to $100 \mathrm{~g}$ by adding $\mathrm{HNO}_{3}$ $(2 \%)$.

Whole-rock $\mathrm{Sr}$ and $\mathrm{Nd}$ isotope measurements were carried out at the Isotope Laboratory of Wuhan Geological Survey Center, China Geological Survey. The Sr and Nd isotopic compositions were analyzed by thermoelectric ionization mass spectrometer using a Triton instrument. Sr and Nd single element solution from Alfa (Alfa Aesar, Karlsruhe, Germany) was used to optimize the instrument operating parameters. An aliquot of the international standard solution of $200 \mu \mathrm{g} \cdot \mathrm{L}^{-1}$ NIST SRM 987 and $200 \mu \mathrm{g} \cdot \mathrm{L}^{-1} \mathrm{JNdi}^{-1}$ were regularly used for evaluating the reproducibility and accuracy of the instrument, respectively. Typically, the signal intensities of ${ }^{88} \mathrm{Sr}$ in NIST SRM 987 were $>$ approximately $4.0 \mathrm{~V}$, and the signal intensities of ${ }^{144} \mathrm{Nd}^{+}$in $\mathrm{JNdi}^{-1}$ were > approximately $2.5 \mathrm{~V}$. The rare earth element (REE) solution from the Sr column was evaporated to incipient dryness and taken up with $0.18 \mathrm{M}$ $\mathrm{HCl}$. The converted REE solution was loaded into an ion-exchange column packed with LN resin. After the completion of draining the sample solution, the columns were rinsed with $0.18 \mathrm{M} \mathrm{HCl}$ to remove undesirable matrix elements. Then, the $\mathrm{Nd}$ fraction was eluted using $0.3 \mathrm{M} \mathrm{HCl}$ and gently evaporated to dryness prior to mass-spectrometric measurement. The $\mathrm{Sr}$ and $\mathrm{Nd}$ isotopic data were acquired in a static mode at low resolution. The routine data acquisition consisted of ten blocks of 10 cycles (4.194 s integration time per cycle). The mass fractionation corrections for $\mathrm{Sr}$ and $\mathrm{Nd}$ isotopic ratios were normalized to 
${ }^{86} \mathrm{Sr} /{ }^{88} \mathrm{Sr}=0.1194,{ }^{146} \mathrm{Nd} /{ }^{144} \mathrm{Nd}=0.7219$, respectively. The detailed analytical procedures can be found in $[37,38]$.

Zircon grains were extracted from four rock samples (two Zengudi and two Tuobake granite porphyries), using electromagnetic selection and flotation at the Langfang Diyan Mineral Separation Company (Hebei, China). About 500 zircon grains were selected from each sample, then handpicked under a binocular microscope, mounted in epoxy resin, and finally polished to expose the grain core. Cathodoluminescence (CL) images of zircon grains were obtained at the Wuhan Sample Solution Analytical Technology Co., Ltd., using a scanning electron microscope coupled with an energy dispersive spectroscopy (EDS) system and a $\mathrm{CL}^{3+}$ detector under operating conditions of $15 \mathrm{kV}$ and $4 \mathrm{nA}$. These CL images were examined for selecting zircon grains without cracks and inclusions, which were further analyzed in the same laboratory by laser ablation-inductively coupled plasma mass spectrometry (LA-ICPMS). Zircon U-Pb dating and trace element analyses were performed using a $193 \mathrm{~nm}$ ArFexcimer (COMPexPro) with laser power of $8 \mathrm{~J} / \mathrm{cm}^{2}$ coupled with an Agilent 7700e ICPMS instrument. The spot size and frequency of the laser were set as $32 \mu \mathrm{m}$ and $5 \mathrm{~Hz}$, respectively. Detailed operating conditions for the laser ablation system and the ICP-MS instrument and data reduction can be found in [39-41]. Zircon 91500 and GJ-1 were used as external standards for $\mathrm{U}-\mathrm{Pb}$ dating and trace element calibration, respectively. Concordia diagrams and weighted mean calculations were performed with Isoplot/Ex_ver3 (Isoplot4 by Kenneth R. Ludwig of American).

Subsequently, in situ Hf isotope measurements were performed on the same zircon grain or spot that was previously analyzed for $\mathrm{U}-\mathrm{Pb}$ dating at the Wuhan Sample Solution Analytical Technology Co., Ltd., Hubei, China. Zircon Lu-Hf isotope analyses were conducted using a Neptune Plus MC-ICP-MS in combination with a Geolas HD excimer laser ablation system. Detailed operating conditions for the laser ablation system and the MC-ICP-MS instrument and analytical method are the same as described by Hu et al. 2012 [42]. All analyzed spots used a beam diameter of $44 \mu \mathrm{m}$ and pulse frequency of $8 \mathrm{~Hz}$ with the GJ-1 zircon standard. The ${ }^{179} \mathrm{Hf} /{ }^{177} \mathrm{Hf}$ and ${ }^{173} \mathrm{Yb} /{ }^{171} \mathrm{Yb}$ ratios were used to calculate the mass bias of $\mathrm{Hf}(\beta \mathrm{Hf})$ and $\mathrm{Yb}(\beta \mathrm{Yb})$, which were normalized to ${ }^{179} \mathrm{Hf} /{ }^{177} \mathrm{Hf}$ $=0.7325$ and ${ }^{173} \mathrm{Yb} /{ }^{171} \mathrm{Yb}=1.132685$ [43] using an exponential correction for mass bias. Interference of ${ }^{176} \mathrm{Yb}$ on ${ }^{176} \mathrm{Hf}$ was corrected by measuring the interference-free ${ }^{173} \mathrm{Yb}$ isotope and using ${ }^{176} \mathrm{Yb} /{ }^{173} \mathrm{Yb}=0.79639$ [43] to calculate ${ }^{176} \mathrm{Yb} /{ }^{177} \mathrm{Hf}$. Similarly, the relatively minor interference of ${ }^{176} \mathrm{Lu}$ on ${ }^{176} \mathrm{Hf}$ was corrected by measuring the intensity of the interference-free ${ }^{175} \mathrm{Lu}$ isotope and using the recommended ${ }^{176} \mathrm{Lu} /{ }^{175} \mathrm{Lu}=0.02656$ [44] to calculate ${ }^{176} \mathrm{Lu} /{ }^{177} \mathrm{Hf}$.

\section{Results}

\subsection{Whole Rock Major and Trace Element Compositions}

The whole rock major and trace element analytical results are shown in Table 1.

Table 1. Major (wt.\%) and trace element (ppm) compositions of the Zengudi and Tuobake granite porphyries. LREE, light rare earth element; $\mathrm{HREE}$, heavy rare earth element; $\mathrm{A} / \mathrm{CNK}, n \mathrm{Al}_{2} \mathrm{O}_{3} /\left(\mathrm{K}_{2} \mathrm{O}+\mathrm{Na}_{2} \mathrm{O}+\mathrm{K}_{2} \mathrm{O}\right)$.

\begin{tabular}{ccccccccccc}
\hline \multirow{2}{*}{ Sample } & \multicolumn{4}{c}{ Zengudi Granite Porphyry } & \multicolumn{4}{c}{ Tuobake Granite Porphyry } \\
\cline { 2 - 10 } & SC-H1 & SC-H2 & SC-H3 & SC-H4 & SC-H5 & YZ-H1 & YZ-H2 & YZ-H3 & YZ-H4 & YZ-H5 \\
\hline $\mathbf{S i O}_{\mathbf{2}}$ & 87.61 & 80.32 & 81.96 & 68.04 & 69.68 & 71.07 & 72.97 & 77.75 & 72.42 & 73.84 \\
$\mathbf{T i O}_{\mathbf{2}}$ & 0.264 & 0.230 & 0.299 & 0.771 & 0.727 & 0.545 & 0.493 & 0.333 & 0.486 & 0.417 \\
$\mathbf{A l}_{\mathbf{2}} \mathbf{O}_{\mathbf{3}}$ & 5.55 & 9.25 & 9.12 & 13.18 & 13.33 & 13.98 & 13.09 & 9.21 & 12.80 & 11.38 \\
$\mathbf{F e}_{\mathbf{2}} \mathbf{O}_{\mathbf{3}}$ & 1.38 & 2.18 & 2.18 & 4.51 & 5.16 & 2.67 & 2.99 & 2.24 & 3.62 & 3.72 \\
$\mathbf{M n O}$ & 0.072 & 0.031 & 0.022 & 0.063 & 0.057 & 0.042 & 0.035 & 0.064 & 0.035 & 0.050 \\
$\mathbf{M g O}$ & 0.42 & 0.97 & 0.91 & 1.54 & 2.10 & 0.68 & 0.68 & 0.38 & 0.74 & 0.70 \\
$\mathbf{C a O}$ & 0.71 & 1.02 & 0.22 & 2.15 & 0.72 & 1.71 & 1.54 & 2.66 & 1.57 & 2.06 \\
$\mathbf{N a}_{\mathbf{2}} \mathbf{O}$ & 0.06 & 1.86 & 2.76 & 2.38 & 3.95 & 1.89 & 1.91 & 2.76 & 1.71 & 1.72 \\
\hline
\end{tabular}


Table 1. Cont.

\begin{tabular}{|c|c|c|c|c|c|c|c|c|c|c|}
\hline \multirow{2}{*}{ Sample } & \multicolumn{5}{|c|}{ Zengudi Granite Porphyry } & \multicolumn{5}{|c|}{ Tuobake Granite Porphyry } \\
\hline & SC-H1 & SC-H2 & SC-H3 & SC-H4 & SC-H5 & YZ-H1 & YZ-H2 & YZ-H3 & YZ-H4 & YZ-H5 \\
\hline $\mathrm{K}_{2} \mathrm{O}$ & 1.48 & 1.97 & 1.60 & 4.11 & 2.52 & 4.16 & 3.62 & 1.63 & 3.48 & 2.96 \\
\hline $\mathrm{P}_{2} \mathrm{O}_{5}$ & 0.043 & 0.069 & 0.076 & 0.157 & 0.160 & 0.171 & 0.156 & 0.106 & 0.143 & 0.115 \\
\hline LOI & 2.39 & 2.03 & 1.13 & 3.17 & 1.95 & 3.01 & 2.84 & 2.99 & 2.91 & 3.13 \\
\hline Total & 99.96 & 99.95 & 100.27 & 100.07 & 100.35 & 99.94 & 100.32 & 100.12 & 99.91 & 100.10 \\
\hline $\mathbf{L i}$ & 55.0 & 9.22 & 7.10 & 11.6 & 16.6 & 14.8 & 13.9 & 8.35 & 14.3 & 13.0 \\
\hline $\mathrm{Be}$ & 0.92 & 1.32 & 1.09 & 1.35 & 2.46 & 3.37 & 3.20 & 1.57 & 3.15 & 2.49 \\
\hline Sc & 3.64 & 4.36 & 5.19 & 10.9 & 11.8 & 8.13 & 7.30 & 4.71 & 7.92 & 6.59 \\
\hline V & 31.7 & 27.5 & 29.9 & 52.1 & 56.8 & 29.6 & 29.0 & 17.6 & 28.9 & 25.5 \\
\hline $\mathrm{Cr}$ & 13.3 & 20.4 & 21.9 & 33.7 & 31.4 & 16.6 & 14.6 & 10.8 & 14.7 & 12.7 \\
\hline Co & 1.94 & 3.61 & 4.35 & 4.76 & 9.19 & 3.62 & 4.19 & 4.33 & 5.16 & 6.09 \\
\hline $\mathbf{N i}$ & 4.94 & 9.09 & 9.14 & 15.0 & 15.7 & 5.71 & 6.56 & 7.14 & 7.75 & 7.70 \\
\hline $\mathrm{Cu}$ & 2.64 & 3.12 & 1.09 & 2.92 & 12.0 & 5.61 & 12.4 & 2.60 & 16.6 & 12.5 \\
\hline $\mathrm{Zn}$ & 7.66 & 22.4 & 21.6 & 41.5 & 46.7 & 31.9 & 36.4 & 26.1 & 43.9 & 73.7 \\
\hline Ga & 7.80 & 11.0 & 10.6 & 16.5 & 18.4 & 19.7 & 19.0 & 9.61 & 19.0 & 16.9 \\
\hline $\mathrm{Rb}$ & 80.7 & 98.0 & 66.0 & 169 & 74.0 & 248 & 224 & 96.2 & 219 & 183 \\
\hline $\mathrm{Sr}$ & 37.4 & 41.3 & 40.6 & 76.8 & 63.3 & 81.5 & 69.9 & 65.4 & 67.1 & 68.2 \\
\hline$Y$ & 13.7 & 16.2 & 20.5 & 34.3 & 40.0 & 51.5 & 48.5 & 35.9 & 44.4 & 43.1 \\
\hline $\mathrm{Zr}$ & 138 & 121 & 151 & 300 & 291 & 289 & 276 & 181 & 254 & 235 \\
\hline $\mathrm{Nb}$ & 6.67 & 6.39 & 7.77 & 15.6 & 14.6 & 15.0 & 13.7 & 9.57 & 13.2 & 11.7 \\
\hline Sn & 2.67 & 2.44 & 2.18 & 4.75 & 4.53 & 6.33 & 5.94 & 3.89 & 5.49 & 4.94 \\
\hline Cs & 6.76 & 2.85 & 2.65 & 1.53 & 1.59 & 9.39 & 9.01 & 2.91 & 8.78 & 7.83 \\
\hline $\mathbf{B a}$ & 157 & 276 & 318 & 765 & 515 & 785 & 594 & 240 & 582 & 546 \\
\hline $\mathrm{La}$ & 21.1 & 15.0 & 37.3 & 20.5 & 43.5 & 51.9 & 49.8 & 32.2 & 46.1 & 40.7 \\
\hline $\mathrm{Ce}$ & 39.8 & 28.7 & 67.5 & 42.2 & 85.9 & 104 & 100 & 64.9 & 91.3 & 82.0 \\
\hline Pr & 4.54 & 3.20 & 6.98 & 4.93 & 9.60 & 11.6 & 11.2 & 7.25 & 10.1 & 9.11 \\
\hline $\mathrm{Nd}$ & 15.9 & 11.9 & 24.0 & 19.5 & 36.0 & 43.7 & 41.5 & 27.9 & 38.1 & 34.2 \\
\hline Sm & 3.02 & 2.45 & 4.21 & 4.68 & 7.61 & 9.56 & 9.23 & 6.02 & 8.52 & 7.25 \\
\hline $\mathrm{Eu}$ & 0.34 & 0.45 & 0.72 & 0.52 & 1.06 & 1.33 & 1.03 & 0.89 & 1.06 & 0.90 \\
\hline $\mathrm{Gd}$ & 2.23 & 2.15 & 3.06 & 4.55 & 6.41 & 8.23 & 7.58 & 5.50 & 7.26 & 6.77 \\
\hline $\mathrm{Tb}$ & 0.35 & 0.44 & 0.54 & 0.84 & 1.13 & 1.43 & 1.33 & 0.95 & 1.26 & 1.21 \\
\hline Dy & 2.39 & 2.55 & 3.41 & 5.38 & 6.93 & 8.65 & 8.36 & 5.79 & 7.59 & 7.26 \\
\hline Ho & 0.46 & 0.56 & 0.66 & 1.15 & 1.38 & 1.73 & 1.65 & 1.15 & 1.53 & 1.43 \\
\hline Er & 1.38 & 1.51 & 1.87 & 3.41 & 3.83 & 4.90 & 4.56 & 3.35 & 4.35 & 4.01 \\
\hline $\mathrm{Tm}$ & 0.23 & 0.23 & 0.30 & 0.54 & 0.59 & 0.71 & 0.71 & 0.50 & 0.63 & 0.62 \\
\hline $\mathrm{Yb}$ & 1.41 & 1.41 & 1.68 & 3.31 & 3.42 & 4.37 & 4.09 & 2.80 & 3.76 & 3.65 \\
\hline $\mathbf{L u}$ & 0.21 & 0.23 & 0.26 & 0.47 & 0.54 & 0.70 & 0.60 & 0.45 & 0.61 & 0.56 \\
\hline Hf & 3.61 & 3.13 & 4.00 & 7.82 & 7.66 & 7.71 & 7.24 & 4.70 & 6.60 & 6.29 \\
\hline Ta & 0.54 & 0.56 & 0.58 & 1.16 & 1.10 & 1.13 & 1.02 & 0.76 & 1.01 & 0.91 \\
\hline $\mathrm{Tl}$ & 0.64 & 0.46 & 0.35 & 0.89 & 0.41 & 1.28 & 1.11 & 0.54 & 1.10 & 0.94 \\
\hline $\mathrm{Pb}$ & 41.0 & 1.82 & 2.05 & 9.12 & 2.11 & 15.0 & 29.3 & 16.9 & 33.5 & 27.8 \\
\hline Th & 11.7 & 12.3 & 16.9 & 24.8 & 23.6 & 29.0 & 26.4 & 19.0 & 25.6 & 23.1 \\
\hline $\mathrm{U}$ & 2.90 & 3.10 & 3.30 & 4.99 & 5.20 & 6.74 & 5.87 & 4.30 & 5.95 & 5.24 \\
\hline$\Sigma$ REE & 93.4 & 70.8 & 152 & 112 & 208 & 252 & 242 & 160 & 222 & 200 \\
\hline LREE & 84.8 & 61.7 & 141 & 92.4 & 184 & 222 & 213 & 139 & 195 & 174 \\
\hline HREE & 8.65 & 9.07 & 11.78 & 19.65 & 24.23 & 30.72 & 28.88 & 20.50 & 26.99 & 25.51 \\
\hline LREE/HREE & 9.80 & 6.80 & 11.94 & 4.70 & 7.58 & 7.22 & 7.37 & 6.79 & 7.24 & 6.83 \\
\hline $\mathrm{La}_{\mathrm{N}} / \mathrm{Yb} \mathrm{b}_{\mathrm{N}}$ & 10.7 & 7.62 & 15.95 & 4.45 & 9.12 & 8.53 & 8.74 & 8.24 & 8.81 & 8.00 \\
\hline $\mathbf{E u} / \mathbf{E u}^{*}$ & 0.40 & 0.59 & 0.61 & 0.35 & 0.47 & 0.46 & 0.38 & 0.47 & 0.41 & 0.39 \\
\hline $\mathrm{Ce} / \mathrm{Ce}^{*}$ & 1.00 & 1.02 & 1.03 & 1.03 & 1.03 & 1.03 & 1.04 & 1.04 & 1.04 & 1.04 \\
\hline $\mathrm{Na}_{2} \mathrm{O}+\mathrm{K}_{2} \mathrm{O}$ & 1.54 & 3.83 & 4.36 & 6.49 & 6.47 & 6.06 & 4.39 & 5.19 & 4.68 & 5.53 \\
\hline $\mathrm{K}_{2} \mathrm{O} / \mathrm{Na}_{2} \mathrm{O}$ & 25.05 & 1.06 & 0.58 & 1.73 & 0.64 & 2.20 & 1.89 & 0.59 & 2.04 & 1.72 \\
\hline A/CNK & 1.86 & 1.31 & 1.37 & 1.07 & 1.27 & 1.30 & 1.33 & 0.83 & 1.36 & 1.16 \\
\hline $\mathrm{Y} / \mathrm{Yb}$ & 9.66 & 11.46 & 12.21 & 10.37 & 11.71 & 11.78 & 11.86 & 12.83 & 11.82 & 11.79 \\
\hline$(\mathrm{Ho} / \mathrm{Yb})_{\mathrm{N}}$ & 10.69 & 7.62 & 15.95 & 4.45 & 9.12 & 8.53 & 8.74 & 8.24 & 8.81 & 8.00 \\
\hline
\end{tabular}

The Zengudi granite porphyry samples have high contents of $\mathrm{SiO}_{2}(68.04-87.61 \%)$, $\mathrm{Al}_{2} \mathrm{O}_{3}(5.55-18.33 \%), \mathrm{Na}_{2} \mathrm{O}(0.06-3.95 \%), \mathrm{K}_{2} \mathrm{O}(1.48-4.11 \%)$, and $\mathrm{Na}_{2} \mathrm{O}+\mathrm{K}_{2} \mathrm{O}(1.54-6.49 \%)$ 
(Figure 3a). The $\mathrm{K}_{2} \mathrm{O} / \mathrm{Na}_{2} \mathrm{O}$ ratios vary between 0.58 and 25.05. Except for sample SC$\mathrm{H} 1$, most of the Zengudi granites plot in the peraluminous field (A/CNK $=1.07-1.86$; Figure $3 b)$. In addition, these granitic rocks contain low contents of $\mathrm{P}_{2} \mathrm{O}_{5}(0.043-0.16 \%)$, $\mathrm{CaO}(0.71-2.15 \%), \mathrm{TiO}_{2}(0.23-0.77 \%)$ and $\mathrm{Fe}_{2} \mathrm{O}_{3}{ }^{\mathrm{T}}$ (1.38-5.16\%). The Zengudi granite porphyries are medium-to-high $\mathrm{K}$ and calc-alkaline rocks (Figure $3 \mathrm{c}, \mathrm{d}$ ). The CIPW standard mineral calculation shows that the contents of corundum range between 1.20 and 3.18, and high differentiation index (DI) from 78.74 to 90.99 . The total rare earth element contents ( $\sum$ REE) of the granite porphyry samples vary from 70.75 to $207.93 \mathrm{ppm}$, with high LREE/HREE ratios of 4.70-11.94. The $\mathrm{La}_{\mathrm{N}} / \mathrm{Yb}_{\mathrm{N}}$ ratios range between 4.45 and 15.95. These granitic rocks show a negative $\mathrm{Eu}$ anomaly with $\mathrm{Eu} / \mathrm{Eu}^{*}$ ratios ranging from 0.35 to 0.61 .
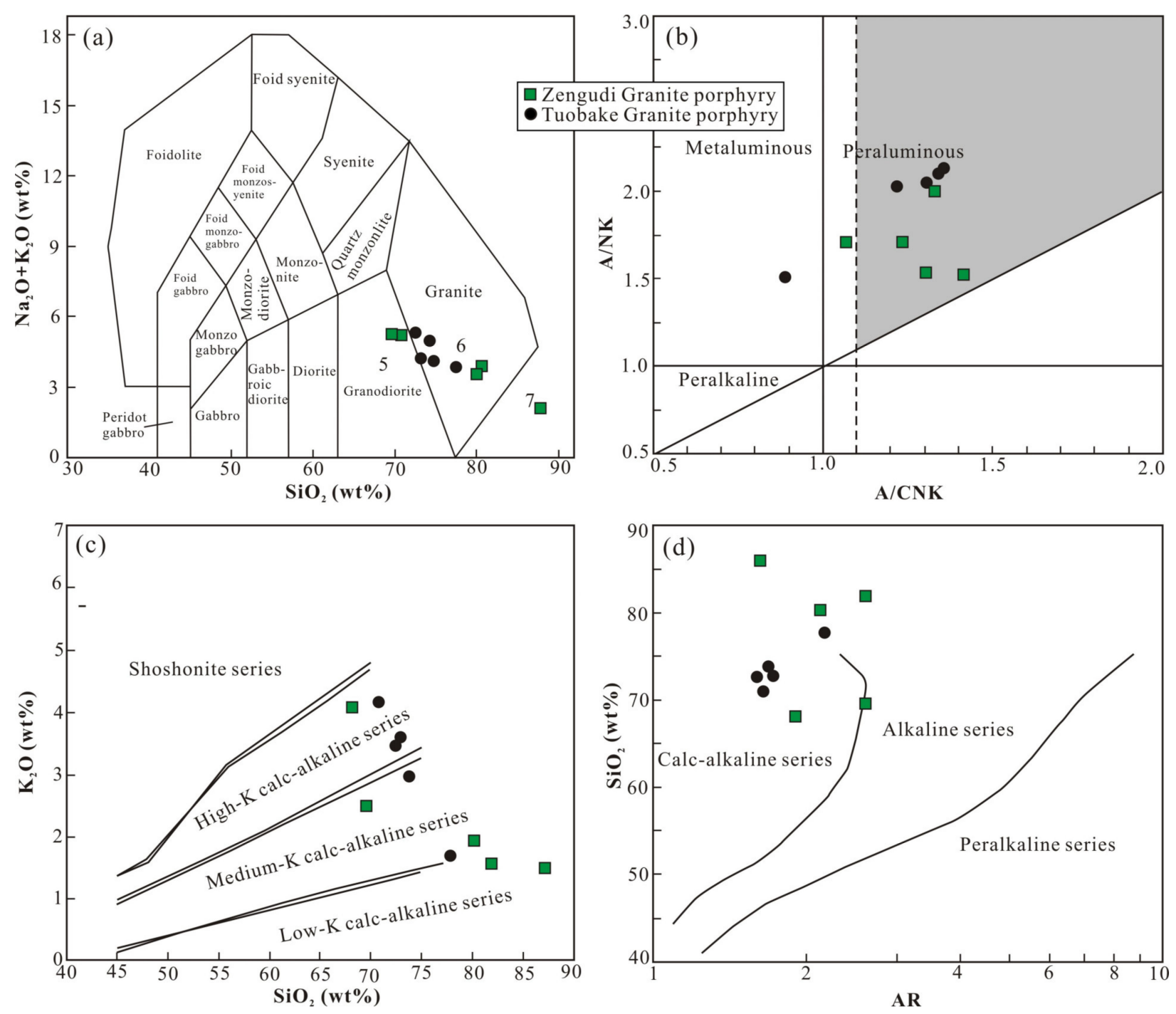

Figure 3. Geochemical classification diagrams of the Zengudi and Tuobake granite porphyries. (a) $\mathrm{SiO}_{2} \mathrm{versus}\left(\mathrm{K}_{2} \mathrm{O}+\right.$ $\mathrm{Na}_{2} \mathrm{O}$ ) [45], (b) A/CNK versus A/NK [46,47], (c) $\mathrm{SiO}_{2}$ versus $\mathrm{K}_{2} \mathrm{O}$ [48], and (d) $\mathrm{SiO}_{2}$ versus AR [49].

The Tuobake granite porphyry samples have high concentrations of $\mathrm{SiO}_{2}(71.07-77.75 \%)$, $\mathrm{Al}_{2} \mathrm{O}_{3}(9.21-13.98 \%)$ and $\mathrm{Na}_{2} \mathrm{O}+\mathrm{K}_{2} \mathrm{O}(4.39-6.06 \%)$, and low contents of $\mathrm{Fe}_{2} \mathrm{O}_{3}{ }^{\mathrm{T}}(2.24-3.72 \%)$ and $\mathrm{P}_{2} \mathrm{O}_{5}(0.106-0.171 \%)$. In the $\mathrm{SiO}_{2}$ versus $\left(\mathrm{K}_{2} \mathrm{O}+\mathrm{Na}_{2} \mathrm{O}\right)$ diagram (Figure $\left.3 a\right)$, all samples plot in the granite field. Except for sample YZ-H3, most of the Tuobake granites plot in the peraluminous field $(\mathrm{A} / \mathrm{CNK}=0.83-1.36$, average $=1.20)$ (Figure $3 \mathrm{~b})$. In the $\mathrm{SiO}_{2} \mathrm{vs} \mathrm{K}_{2} \mathrm{O}$ diagram (Figure 3c), these granite samples plot in the field of low-to-high $\mathrm{K}$ calc-alkaline series, whereas they mostly cluster within the field of calc-alkaline series in the diagram of 
$\mathrm{SiO}_{2}$ versus $\mathrm{AR}\left(\left(\mathrm{Al}_{2} \mathrm{O}_{3}+\mathrm{CaO}+\mathrm{Na}_{2} \mathrm{O}+\mathrm{K}_{2} \mathrm{O}\right) /\left(\mathrm{Al}_{2} \mathrm{O}_{3}+\mathrm{CaO}-\left(\mathrm{Na}_{2} \mathrm{O}+\mathrm{K}_{2} \mathrm{O}\right)\right)\right.$ (Figure 3d). In both diagrams (Figure $3 a, b)$, the Tuobake granites exhibit high silica and aluminum contents. According to the CIPW standard mineral calculation, the contents of corundum vary between 1.88 and 3.79. The $\sum$ REE contents of these granite porphyry samples range from 159.62 to $241.79 \mathrm{ppm}$, with high LREE/HREE ratios of 6.79-7.37. These granitic rocks display $\mathrm{La}_{\mathrm{N}} / \mathrm{Yb}_{\mathrm{N}}$ ratios ranging from 8.00 to 8.81, and negative Eu anomaly with Eu/Eu* ratios of $0.38-0.47$.

The chondrite-normalized REE patterns of the Zengudi and Tuobake granite porphyries show LREE enrichment and obvious negative Eu anomalies (Figure 4a). The primitive mantle-normalized trace element spider diagram shows that these rocks are significantly depleted in $\mathrm{Sr}, \mathrm{Ba}$ and high field strength elements (HFSE such as $\mathrm{Ta}, \mathrm{Nb}$, and Ti), but enriched in large ion lithophile elements (LILE such as $\mathrm{Rb}, \mathrm{U}, \mathrm{K}$, and Th) (Figure 4b).
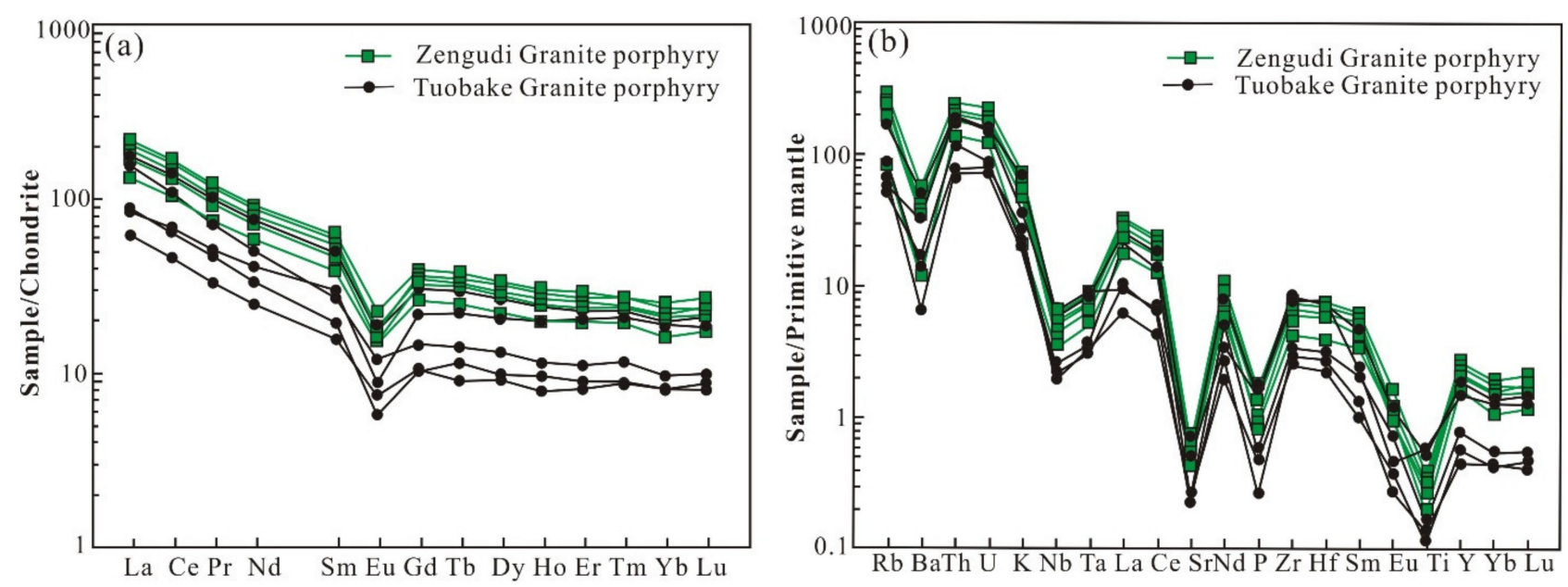

Figure 4. (a) Chondrite-normalized REE patterns and (b) primitive mantle-normalized trace element spider diagram of the Zengudi and Tuobake granite porphyries. The normalized values are from [50].

\subsection{Whole Rock $\mathrm{Sr}-\mathrm{Nd}$ Isotopes}

The whole rock Sr-Nd isotopic data of the studied granite porphyries is presented in Table 2. All the Zengudi granite porphyry samples have low contents of $\mathrm{Rb}(80-110 \mathrm{ppm})$ and $\mathrm{Sr}(68-92 \mathrm{ppm})$, but high initial ${ }^{87} \mathrm{Sr} /{ }^{86} \mathrm{Sr}$ ratios $(0.708462-0.715748$, average $=0.7115980)$. The initial ${ }^{143} \mathrm{Nd} /{ }^{144} \mathrm{Nd}$ ratios range between 0.511574 and 0.511837 (average $=0.511742$ ) and the $\varepsilon_{\mathrm{Nd}}(\mathrm{t})$ values are low $(-14.49$ to -9.35 , average $=-11.21)$. The calculated $\mathrm{Nd}$ model ages $\left(\mathrm{T}_{\mathrm{DM} 2}\right)$ of the Zengudi granite porphyries vary from 1.78 to $2.46 \mathrm{Ga}$.

The Tuobake granite porphyry samples have relatively high contents of $\mathrm{Rb}(190-240 \mathrm{ppm})$ but low contents of $\mathrm{Sr}(66-83 \mathrm{ppm})$. These granite porphyry samples display relatively high initial ${ }^{87} \mathrm{Sr} /{ }^{86} \mathrm{Sr}$ ratios $(0.7160803-0.7309865$, average $=0.720591)$, low initial ${ }^{143} \mathrm{Nd} /{ }^{144} \mathrm{Nd}$ ratios $(0.511763-0.511839$, average $=0.511785)$ and low $\varepsilon_{\mathrm{Nd}}(\mathrm{t})$ values $(-10.9$ to -9.41 , average $=-10.47)$. The calculated $\mathrm{Nd}$ model ages $\left(\mathrm{T}_{\mathrm{DM} 2}\right)$ of the Tuobake granite porphyries range from 1.79 to $1.90 \mathrm{Ga}$. 
Table 2. Whole rock Sr-Nd isotopic compositions of the Zengudi and Tuobake granite porphyries.

\begin{tabular}{|c|c|c|c|c|c|c|c|c|c|c|c|c|c|c|}
\hline $\begin{array}{l}\text { Sample } \\
\text { No. }\end{array}$ & Location & $\begin{array}{l}\text { Age } \\
\text { (Ma) }\end{array}$ & $\begin{array}{c}\mathbf{R b} \\
(\mathrm{ppm})\end{array}$ & $\begin{array}{c}\mathrm{Sr} \\
(\mathrm{ppm})\end{array}$ & $\underset{(\mathrm{ppm})}{\mathrm{Sm}}$ & $\underset{(\mathrm{ppm})}{\mathrm{Nd}}$ & ${ }^{87} \mathrm{Sr} /{ }^{86} \mathrm{Sr}$ & $2 \sigma$ & $\left({ }^{87} \mathrm{Sr} /{ }^{86} \mathrm{Sr}\right)_{i}$ & ${ }^{143} \mathrm{Nd} /{ }^{144} \mathrm{Nd}$ & $2 \sigma$ & $\left({ }^{143} \mathrm{Nd} /{ }^{144} \mathrm{Nd}\right)_{i}$ & $\underset{(t)}{\varepsilon N d}$ & $\begin{array}{l}\mathrm{T}_{\mathrm{DM}} \\
\text { (Ma) }\end{array}$ \\
\hline SC-Y1 & Zengudi & 254 & 170.0 & 75.0 & 3.02 & 15.9 & 0.723590 & 0.00005 & 0.7024524 & 0.51201 & 0.00002 & 0.511822 & -9.65 & 1980 \\
\hline SC-Y2 & Zengudi & 254 & 157.0 & 56.0 & 4.21 & 11.9 & 0.728050 & 0.00003 & 0.7033351 & 0.51196 & 0.00006 & 0.511609 & -13.8 & 2050 \\
\hline SC-Y3 & Zengudi & 254 & 98.0 & 47.0 & 4.68 & 24.0 & 0.722480 & 0.00003 & 0.7040523 & 0.511928 & 0.00004 & 0.511735 & -11.34 & 1944 \\
\hline SC-Y4 & Zengudi & 254 & 135.0 & 61.0 & 7.61 & 19.5 & 0.721900 & 0.00003 & 0.7092388 & 0.511932 & 0.00005 & 0.511547 & -15.01 & 2313 \\
\hline YZ-Y1 & Tuobake & 246 & 240.0 & 83.0 & 9.23 & 43.7 & 0.745380 & 0.00005 & 0.7160803 & 0.511976 & 0.00005 & 0.51177 & -10.76 & 1892 \\
\hline YZ-Y2 & Tuobake & 246 & 223.0 & 71.0 & 6.02 & 41.5 & 0.748340 & 0.00002 & 0.7165142 & 0.51198 & 0.00002 & 0.511839 & -9.41 & 1787 \\
\hline YZ-Y3 & Tuobake & 246 & 215.0 & 66.0 & 8.52 & 38.1 & 0.751790 & 0.00002 & 0.7187814 & 0.51198 & 0.00003 & 0.511763 & -10.9 & 1903 \\
\hline YZ-Y4 & Tuobake & 246 & 190.0 & 70.0 & 7.25 & 34.2 & 0.758490 & 0.00003 & 0.7309865 & 0.511974 & 0.00003 & 0.511768 & -10.8 & 1896 \\
\hline
\end{tabular}

\subsection{Zircon CL Images}

Zircon CL images are presented in Figure 5. All the zircons from the Zengudi (samples SC1801 and SC1802) and Tuobake (samples YZ1803 and YZ1804) granite porphyries show similar morphology. They mostly show euhedral prismatic grains with a length of 80-120 $\mu \mathrm{m}$ and length-to-width ratios between 1:1 and 3:1. All the zircon grains display clear oscillatory zoning, suggesting a magmatic origin [51-54].

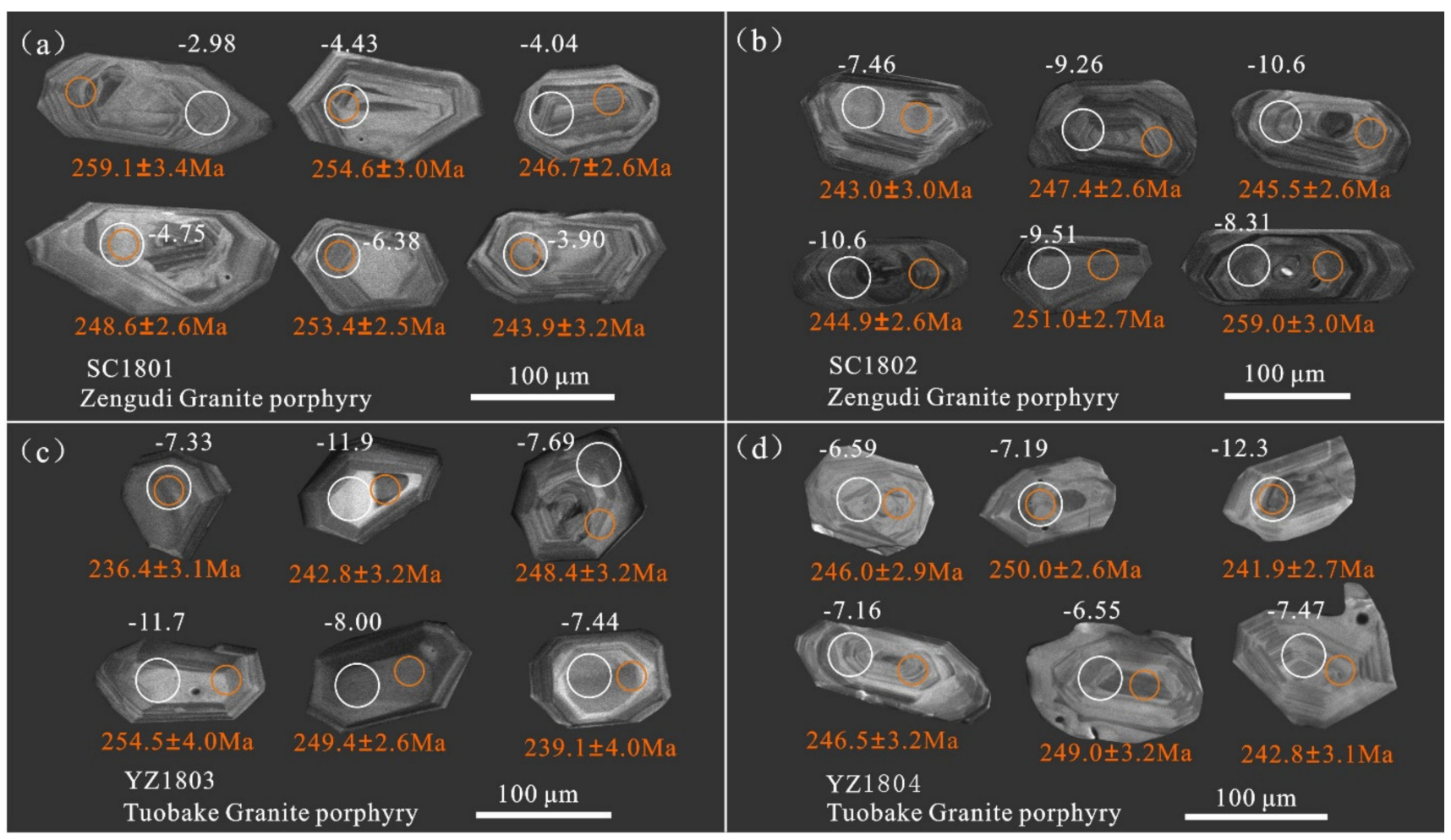

Figure 5. Zircon CL images of representative zircon grains for $(\mathbf{a}, \mathbf{b})$ the Zengudi granite porphyry and $(\mathbf{c}, \mathbf{d})$ the Tuobake granite porphyry. The orange and white circles are the sites for in situ U-Pb isotope dating and Hf isotope analyses, respectively.

\subsection{Zircon $\mathrm{U}-\mathrm{Pb}$ Ages}

The zircon $\mathrm{U}-\mathrm{Pb}$ dating results for the four granite porphyry samples are shown in Table 3. Zircons from the Zengudi granite porphyries (samples SC1801 and SC1802) have $\mathrm{Th} / \mathrm{U}$ ratios $=0.12-0.52$ (average $=0.35)$, which are comparable to those of typical magmatic zircons [55]. The ${ }^{206} \mathrm{~Pb} /{ }^{238} \mathrm{U}$ ages of twenty-four zircon grains from sample SC1801 range between 243.9 $\mathrm{Ma}$ and $261.7 \mathrm{Ma}$, giving a weighted mean age of $253.8 \pm 2.2 \mathrm{Ma}$ $(\mathrm{MSWD}=4.4)$ (Figure 6a). One zircon from this sample has a ${ }^{206} \mathrm{~Pb} /{ }^{238} \mathrm{U}$ age of $462.8 \mathrm{Ma}$ (Table 1), which is regarded as the age of inherited zircon grain. Twenty-five zircon grains from sample SC1802 yield ${ }^{206} \mathrm{~Pb} /{ }^{238} \mathrm{U}$ ages of 242.9 to $259.0 \mathrm{Ma}$, with a weighted mean age of $246.6 \pm 0.54 \mathrm{Ma}(\mathrm{MSWD}=5.3)$ (Figure $6 \mathrm{~b})$. Overall, the crystallization age of the Zengudi granite porphyries is estimated at 254-247 Ma. 

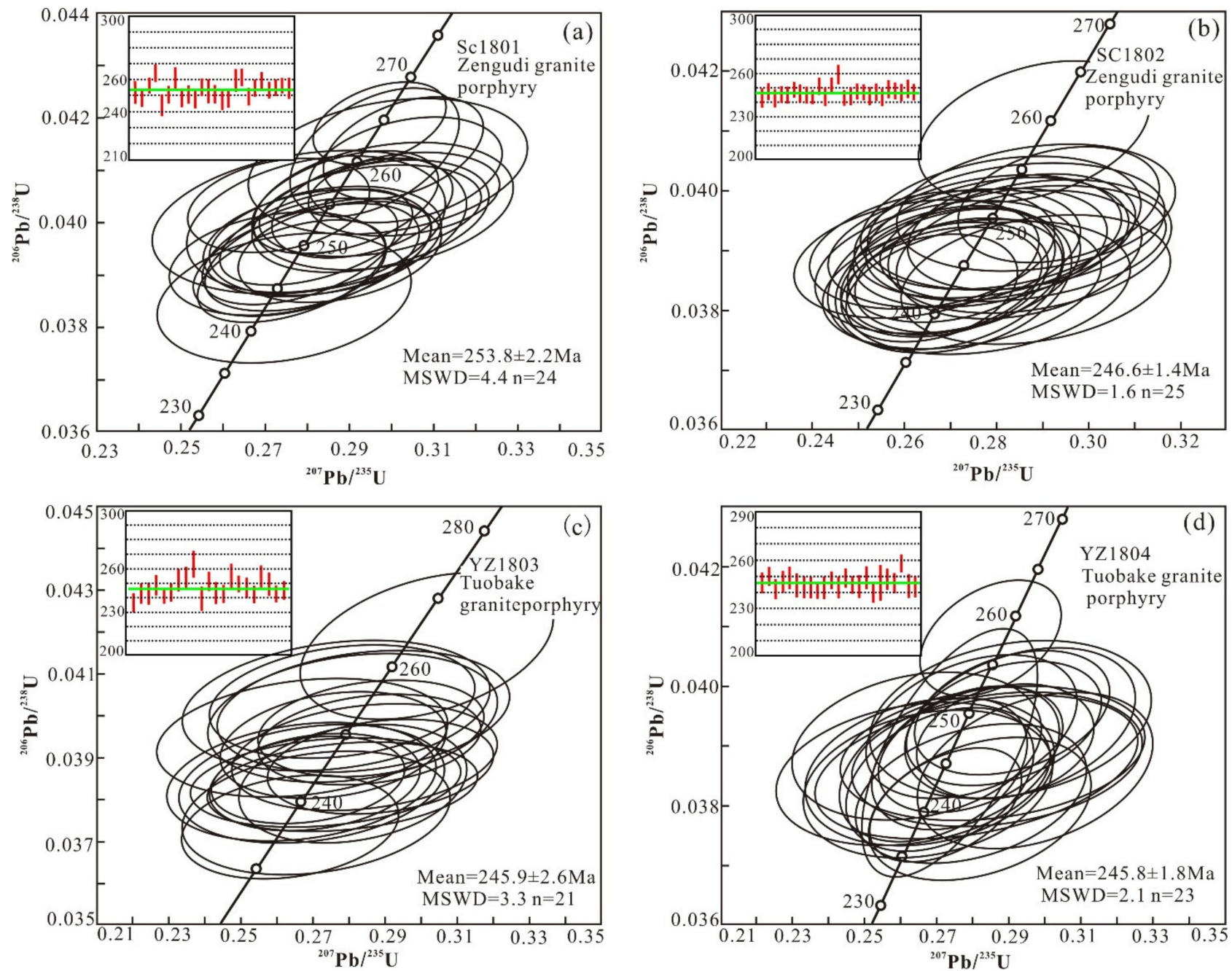

Figure 6. Concordia diagrams for the zircon U-Pb dating data of $(\mathbf{a}, \mathbf{b})$ the Zengudi granite porphyry and (c,d) the Tuobake granite porphyry.

Zircons from the Tuobake granite porphyries (samples YZ1803 and YZ1804) have relatively high $\mathrm{Th} / \mathrm{U}$ ratios $=0.15-0.69$ (average $=0.48$ ), which are similar to those of typical magmatic zircons [53]. The ${ }^{206} \mathrm{~Pb} /{ }^{238} \mathrm{U}$ ages of twenty-five zircon grains from sample YZ1803 vary between 236.4 and $263.2 \mathrm{Ma}$, with a weighted mean age of $245.9 \pm 2.6 \mathrm{Ma}$ $\left(\mathrm{MSWD}=3.3\right.$ ) (Figure 6c). One zircon from this sample yields a ${ }^{206} \mathrm{~Pb} /{ }^{238} \mathrm{U}$ age of $430.8 \mathrm{Ma}$ (Table 1), which is considered as the age of inherited zircon grain. For the sample YZ1804, twenty-three zircon grains yield $\mathrm{a}^{206} \mathrm{~Pb} /{ }^{238} \mathrm{U}$ age varying from 241.1 to $257.8 \mathrm{Ma}$, with a weighted mean age of $245.8 \pm 1.8 \mathrm{Ma}(\mathrm{MSWD}=2.1$ ) (Figure $6 \mathrm{~d}$ ). Consequently, the crystallization age of the Tuobake granite porphyries is estimated at $246 \mathrm{Ma}$, similar to that of the Zengudi granite porphyries. 
Table 3. LA-ICPMS zircon U-Pb analytical results of the Zengudi and Tuobake granite porphyries.

\begin{tabular}{|c|c|c|c|c|c|c|c|c|c|c|c|c|c|c|c|c|}
\hline \multirow{2}{*}{ Spot No. } & \multicolumn{3}{|c|}{ Contents (ppm) } & \multirow{2}{*}{$\mathrm{Th} / \mathrm{U}$} & \multicolumn{6}{|c|}{ Isotope Ratios } & \multicolumn{6}{|c|}{ Age (Ma) } \\
\hline & $\mathrm{Pb}$ & Th & $\mathbf{U}$ & & ${ }^{207} \mathrm{~Pb} /{ }^{235} \mathrm{U}$ & $1 \sigma$ & ${ }^{206} \mathrm{~Pb} /{ }^{238} \mathrm{U}$ & $1 \sigma$ & ${ }^{207} \mathrm{~Pb} /{ }^{206} \mathrm{~Pb}$ & $1 \sigma$ & ${ }^{207} \mathrm{~Pb} /{ }^{235} \mathrm{U}$ & $1 \sigma$ & ${ }^{206} \mathrm{~Pb} /{ }^{238} \mathrm{U}$ & $1 \sigma$ & ${ }^{207} \mathrm{~Pb} /{ }^{206} \mathrm{~Pb}$ & $1 \sigma$ \\
\hline \multicolumn{17}{|c|}{ SC1801, Zengudi Granite Porphyry } \\
\hline SC1801-02 & 31.22 & 289 & 680 & 0.42 & 0.3032 & 0.0094 & 0.0410 & 0.0004 & 0.0534 & 0.0015 & 269 & 7.3 & 259.1 & 2.6 & 346 & 64.8 \\
\hline SC1801-03 & 27.95 & 140 & 648 & 0.22 & 0.3030 & 0.0093 & 0.0405 & 0.0003 & 0.0541 & 0.0017 & 269 & 7.3 & 256.2 & 2.0 & 376 & 68.5 \\
\hline SC1801-04 & 10.05 & 91.7 & 224 & 0.41 & 0.2850 & 0.0138 & 0.0399 & 0.0005 & 0.0524 & 0.0026 & 255 & 10.9 & 252.1 & 3.4 & 302 & 111 \\
\hline SC1801-05 & 11.39 & 90.2 & 253 & 0.36 & 0.2990 & 0.0136 & 0.0411 & 0.0005 & 0.0532 & 0.0025 & 266 & 10.6 & 259.5 & 3.2 & 345 & 103 \\
\hline SC1801-07 & 14.13 & 146 & 309 & 0.47 & 0.2982 & 0.0125 & 0.0403 & 0.0005 & 0.0536 & 0.0022 & 265 & 9.8 & 254.6 & 3.0 & 354 & 99.1 \\
\hline SC1801-08 & 21.87 & 246 & 479 & 0.51 & 0.2772 & 0.0092 & 0.0393 & 0.0004 & 0.0512 & 0.0018 & 248 & 7.3 & 248.6 & 2.6 & 256 & 77.8 \\
\hline SC1801-09 & 19.20 & 205 & 417 & 0.49 & 0.2949 & 0.0115 & 0.0401 & 0.0004 & 0.0537 & 0.0022 & 262 & 9.0 & 253.4 & 2.5 & 367 & 94.4 \\
\hline SC1801-10 & 22.06 & 132 & 517 & 0.26 & 0.2878 & 0.0098 & 0.0394 & 0.0004 & 0.0529 & 0.0018 & 257 & 7.8 & 249.3 & 2.4 & 324 & 77.8 \\
\hline SC1801-11 & 36.46 & 273 & 824 & 0.33 & 0.2890 & 0.0091 & 0.0406 & 0.0004 & 0.0516 & 0.0016 & 258 & 7.2 & 256.4 & 2.2 & 333 & 72.2 \\
\hline SC1801-12 & 19.66 & 212 & 431 & 0.49 & 0.2855 & 0.0108 & 0.0397 & 0.0004 & 0.0520 & 0.0020 & 255 & 8.5 & 250.7 & 2.6 & 287 & 87.0 \\
\hline SC1801-13 & 18.39 & 210 & 407 & 0.52 & 0.2795 & 0.0106 & 0.0390 & 0.0004 & 0.0520 & 0.0020 & 250 & 8.4 & 246.7 & 2.6 & 283 & 87.0 \\
\hline SC1801-14 & 104.4 & 795 & 2261 & 0.35 & 0.2971 & 0.0065 & 0.0419 & 0.0003 & 0.0512 & 0.0011 & 264 & 5.1 & 264.6 & 2.0 & 250 & 51.8 \\
\hline SC1801-15 & 10.76 & 120 & 244 & 0.49 & 0.2746 & 0.0124 & 0.0386 & 0.0005 & 0.0518 & 0.0023 & 246 & 9.8 & 243.9 & 3.2 & 276 & 104 \\
\hline SC1801-17 & 40.23 & 310 & 881 & 0.35 & 0.2960 & 0.0082 & 0.0413 & 0.0005 & 0.0519 & 0.0015 & 263 & 6.4 & 261.2 & 3.1 & 280 & 64.8 \\
\hline SC1801-18 & 19.88 & 276 & 423 & 0.65 & 0.2803 & 0.0106 & 0.0393 & 0.0004 & 0.0516 & 0.0020 & 251 & 8.4 & 248.7 & 2.7 & 333 & 88.9 \\
\hline SC1801-19 & 26.98 & 252 & 594 & 0.42 & 0.2731 & 0.0088 & 0.0404 & 0.0004 & 0.0488 & 0.0016 & 245 & 7.0 & 255.0 & 2.3 & 139 & 77.8 \\
\hline SC1801-20 & 26.82 & 224 & 593 & 0.38 & 0.2930 & 0.0087 & 0.0403 & 0.0004 & 0.0526 & 0.0016 & 261 & 6.9 & 254.6 & 2.4 & 322 & 70.4 \\
\hline SC1801-21 & 25.10 & 164 & 580 & 0.28 & 0.2781 & 0.0094 & 0.0396 & 0.0004 & 0.0508 & 0.0018 & 249 & 7.5 & 250.6 & 2.4 & 232 & 88.0 \\
\hline SC1801-22 & 26.18 & 212 & 595 & 0.36 & 0.2772 & 0.0087 & 0.0392 & 0.0004 & 0.0514 & 0.0017 & 248 & 7.0 & 247.8 & 2.5 & 257 & 77.8 \\
\hline SC1801-23 & 20.08 & 204 & 445 & 0.46 & 0.2719 & 0.0101 & 0.0392 & 0.0004 & 0.0503 & 0.0020 & 244 & 8.0 & 248.0 & 2.5 & 209 & 90.7 \\
\hline SC1801-24 & 32.77 & 209 & 728 & 0.29 & 0.2882 & 0.0088 & 0.0404 & 0.0004 & 0.0516 & 0.0017 & 257 & 7.0 & 255.3 & 2.4 & 333 & 74.1 \\
\hline SC1801-25 & 7.89 & 66.1 & 173 & 0.38 & 0.2805 & 0.0154 & 0.0400 & 0.0005 & 0.0514 & 0.0031 & 251 & 12.2 & 252.9 & 3.4 & 257 & 132 \\
\hline \multicolumn{17}{|c|}{ SC1802, Zengudi Granite Porphyry } \\
\hline SC1802-01 & 12.22 & 73.4 & 287 & 0.26 & 0.2743 & 0.0115 & 0.0384 & 0.0005 & 0.0515 & 0.0021 & 246 & 9.2 & 243.0 & 3.0 & 265 & 99.1 \\
\hline SC1802-02 & 14.15 & 141 & 315 & 0.45 & 0.2819 & 0.0111 & 0.0391 & 0.0004 & 0.0522 & 0.0021 & 252 & 8.8 & 247.4 & 2.6 & 295 & 94.4 \\
\hline SC1802-03 & 11.95 & 73.5 & 285 & 0.26 & 0.2763 & 0.0134 & 0.0384 & 0.0005 & 0.0522 & 0.0026 & 248 & 10.7 & 242.7 & 2.8 & 295 & 108 \\
\hline SC1802-04 & 13.42 & 82.5 & 317 & 0.26 & 0.2648 & 0.0117 & 0.0388 & 0.0004 & 0.0494 & 0.0022 & 239 & 9.4 & 245.5 & 2.5 & 165 & 138 \\
\hline SC1802-05 & 20.88 & 151 & 492 & 0.31 & 0.2650 & 0.0099 & 0.0387 & 0.0004 & 0.0493 & 0.0018 & 239 & 8.0 & 244.9 & 2.6 & 161 & 91.7 \\
\hline SC1802-06 & 21.53 & 147 & 497 & 0.30 & 0.2855 & 0.0102 & 0.0393 & 0.0004 & 0.0527 & 0.0020 & 255 & 8.0 & 248.6 & 2.5 & 317 & 85.2 \\
\hline
\end{tabular}


Table 3. Cont.

\begin{tabular}{|c|c|c|c|c|c|c|c|c|c|c|c|c|c|c|c|c|}
\hline \multirow{2}{*}{ Spot No. } & \multicolumn{3}{|c|}{ Contents (ppm) } & \multirow{2}{*}{$\mathrm{Th} / \mathrm{U}$} & \multicolumn{6}{|c|}{ Isotope Ratios } & \multicolumn{6}{|c|}{ Age (Ma) } \\
\hline & $\mathbf{P b}$ & Th & $\mathrm{U}$ & & ${ }^{207} \mathrm{~Pb} /{ }^{235} \mathrm{U}$ & $1 \sigma$ & ${ }^{206} \mathrm{~Pb} /{ }^{238} \mathrm{U}$ & $1 \sigma$ & ${ }^{207} \mathrm{~Pb} /{ }^{206} \mathrm{~Pb}$ & $1 \sigma$ & ${ }^{207} \mathrm{~Pb} /{ }^{235} \mathrm{U}$ & $1 \sigma$ & ${ }^{206} \mathrm{~Pb} /{ }^{238} \mathrm{U}$ & $1 \sigma$ & ${ }^{207} \mathrm{~Pb} /{ }^{206} \mathrm{~Pb}$ & $1 \sigma$ \\
\hline SC1802-07 & 17.47 & 117 & 411 & 0.28 & 0.2850 & 0.0108 & 0.0389 & 0.0004 & 0.0530 & 0.0021 & 255 & 8.6 & 246.2 & 2.7 & 332 & 88.9 \\
\hline SC1802-08 & 20.30 & 129 & 481 & 0.27 & 0.2732 & 0.0102 & 0.0388 & 0.0004 & 0.0509 & 0.0019 & 245 & 8.1 & 245.1 & 2.6 & 239 & 88.9 \\
\hline SC1802-09 & 25.03 & 159 & 598 & 0.27 & 0.2695 & 0.0083 & 0.0385 & 0.0003 & 0.0505 & 0.0016 & 242 & 6.7 & 243.7 & 2.1 & 220 & 78.7 \\
\hline SC1802-10 & 12.19 & 73.6 & 281 & 0.26 & 0.2886 & 0.0125 & 0.0397 & 0.0004 & 0.0526 & 0.0024 & 257 & 9.9 & 251.0 & 2.7 & 309 & 99 \\
\hline SC1802-11 & 13.99 & 91.2 & 336 & 0.27 & 0.2694 & 0.0100 & 0.0385 & 0.0005 & 0.0505 & 0.0019 & 242 & 8.0 & 243.6 & 2.9 & 220 & 87.0 \\
\hline SC1802-12 & 23.82 & 66.8 & 572 & 0.12 & 0.2935 & 0.0089 & 0.0398 & 0.0004 & 0.0532 & 0.0017 & 261 & 7.0 & 251.4 & 2.5 & 345 & 72.2 \\
\hline SC1802-13 & 19.11 & 153 & 416 & 0.37 & 0.2883 & 0.0102 & 0.0410 & 0.0005 & 0.0508 & 0.0019 & 257 & 8.1 & 259.0 & 3.0 & 235 & 85.2 \\
\hline SC1802-14 & 21.89 & 156 & 513 & 0.30 & 0.2629 & 0.0084 & 0.0384 & 0.0004 & 0.0494 & 0.0016 & 237 & 6.8 & 242.9 & 2.3 & 165 & 77.8 \\
\hline SC1802-16 & 13.88 & 91.7 & 328 & 0.28 & 0.2736 & 0.0109 & 0.0386 & 0.0004 & 0.0513 & 0.0021 & 246 & 8.7 & 244.1 & 2.6 & 254 & 88.0 \\
\hline SC1802-17 & 20.70 & 138 & 485 & 0.28 & 0.2778 & 0.0106 & 0.0391 & 0.0004 & 0.0512 & 0.0019 & 249 & 8.5 & 247.3 & 2.5 & 250 & 91.7 \\
\hline SC1802-18 & 23.04 & 169 & 534 & 0.32 & 0.2736 & 0.0100 & 0.0390 & 0.0004 & 0.0509 & 0.0019 & 246 & 7.9 & 246.8 & 2.4 & 235 & 87.0 \\
\hline SC1802-19 & 15.49 & 121 & 361 & 0.33 & 0.2728 & 0.0109 & 0.0386 & 0.0004 & 0.0516 & 0.0022 & 245 & 8.7 & 244.1 & 2.8 & 265 & 101 \\
\hline SC1802-20 & 13.39 & 89.5 & 314 & 0.28 & 0.2872 & 0.0125 & 0.0390 & 0.0004 & 0.0535 & 0.0024 & 256 & 9.8 & 246.9 & 2.7 & 350 & 100.0 \\
\hline SC1802-21 & 13.26 & 98.0 & 311 & 0.32 & 0.2885 & 0.0120 & 0.0385 & 0.0005 & 0.0546 & 0.0023 & 257 & 9.4 & 243.6 & 2.8 & 394 & 94.4 \\
\hline SC1802-22 & 13.22 & 74.7 & 310 & 0.24 & 0.2844 & 0.0131 & 0.0394 & 0.0004 & 0.0525 & 0.0025 & 254 & 10.4 & 249.0 & 2.7 & 309 & 107 \\
\hline SC1802-23 & 10.98 & 83.8 & 253 & 0.33 & 0.2782 & 0.0118 & 0.0392 & 0.0004 & 0.0519 & 0.0024 & 249 & 9.4 & 247.8 & 2.6 & 280 & 106 \\
\hline SC1802-24 & 20.32 & 152 & 474 & 0.32 & 0.2680 & 0.0100 & 0.0390 & 0.0004 & 0.0500 & 0.0019 & 241 & 8.0 & 246.6 & 2.6 & 195 & 90.7 \\
\hline SC1802-26 & 17.47 & 113 & 411 & 0.27 & 0.2642 & 0.0102 & 0.0391 & 0.0004 & 0.0490 & 0.0019 & 238 & 8.2 & 247.0 & 2.4 & 146 & 90.7 \\
\hline \multicolumn{17}{|c|}{ YZ1803, Tuobake Granite Porphyry } \\
\hline YZ1803-01 & 10.99 & 93.9 & 229 & 0.41 & 0.3016 & 0.0144 & 0.0417 & 0.0007 & 0.0533 & 0.0029 & 268 & 11.2 & 263.2 & 4.4 & 339 & 122 \\
\hline YZ1803-02 & 11.91 & 131 & 268 & 0.49 & 0.2637 & 0.0122 & 0.0374 & 0.0005 & 0.0514 & 0.0025 & 238 & 9.8 & 236.4 & 3.1 & 261 & 113 \\
\hline YZ1803-03 & 10.48 & 128 & 227 & 0.57 & 0.2698 & 0.0119 & 0.0384 & 0.0005 & 0.0511 & 0.0023 & 243 & 9.5 & 242.8 & 3.2 & 256 & 73.1 \\
\hline YZ1803-04 & 7.84 & 95.6 & 172 & 0.56 & 0.2695 & 0.0150 & 0.0384 & 0.0006 & 0.0507 & 0.0029 & 242 & 12.0 & 242.9 & 3.5 & 233 & 133 \\
\hline YZ1803-05 & 10.73 & 120 & 236 & 0.51 & 0.2753 & 0.0128 & 0.0393 & 0.0005 & 0.0508 & 0.0024 & 247 & 10.2 & 248.4 & 3.2 & 232 & 114 \\
\hline YZ1803-06 & 6.58 & 76.0 & 138 & 0.55 & 0.2764 & 0.0174 & 0.0403 & 0.0007 & 0.0502 & 0.0032 & 248 & 13.8 & 254.5 & 4.0 & 211 & 145 \\
\hline YZ1803-07 & 13.56 & 141 & 299 & 0.47 & 0.2854 & 0.0124 & 0.0395 & 0.0004 & 0.0523 & 0.0023 & 255 & 9.8 & 249.5 & 2.6 & 298 & 102 \\
\hline YZ1803-08 & 6.32 & 72.9 & 137 & 0.53 & 0.2670 & 0.0164 & 0.0394 & 0.0006 & 0.0497 & 0.0033 & 240 & 13.2 & 249.3 & 3.7 & 189 & 154 \\
\hline YZ1803-11 & 8.41 & 116 & 178 & 0.65 & 0.2842 & 0.0149 & 0.0390 & 0.0005 & 0.0527 & 0.0028 & 254 & 11.8 & 246.6 & 3.2 & 317 & 122 \\
\hline YZ1803-12 & 13.55 & 113 & 312 & 0.36 & 0.2766 & 0.0112 & 0.0383 & 0.0004 & 0.0521 & 0.0021 & 248 & 8.9 & 242.2 & 2.8 & 300 & 95.4 \\
\hline YZ1803-13 & 6.76 & 97.4 & 147 & 0.66 & 0.2703 & 0.0150 & 0.0378 & 0.0006 & 0.0527 & 0.0032 & 243 & 12.0 & 239.1 & 4.0 & 317 & 139 \\
\hline YZ1803-15 & 13.39 & 157 & 284 & 0.55 & 0.2858 & 0.0127 & 0.0397 & 0.0005 & 0.0523 & 0.0025 & 255 & 10.0 & 251.2 & 2.9 & 298 & 109 \\
\hline YZ1803-16 & 7.69 & 64.9 & 174 & 0.37 & 0.2668 & 0.0152 & 0.0385 & 0.0005 & 0.0502 & 0.0028 & 240 & 12.2 & 243.3 & 3.4 & 206 & 128 \\
\hline YZ1803-18 & 19.47 & 286 & 414 & 0.69 & 0.2654 & 0.0096 & 0.0380 & 0.0004 & 0.0505 & 0.0019 & 239 & 7.7 & 240.7 & 2.4 & 217 & 87.0 \\
\hline
\end{tabular}


Table 3. Cont.

\begin{tabular}{|c|c|c|c|c|c|c|c|c|c|c|c|c|c|c|c|c|}
\hline \multirow{2}{*}{ Spot No. } & \multicolumn{3}{|c|}{ Contents (ppm) } & \multirow{2}{*}{$\mathrm{Th} / \mathrm{U}$} & \multicolumn{6}{|c|}{ Isotope Ratios } & \multicolumn{6}{|c|}{ Age (Ma) } \\
\hline & $\mathrm{Pb}$ & Th & $\mathrm{U}$ & & ${ }^{207} \mathrm{~Pb} /{ }^{235} \mathrm{U}$ & $1 \sigma$ & ${ }^{206} \mathrm{~Pb} /{ }^{238} \mathrm{U}$ & $1 \sigma$ & ${ }^{207} \mathrm{~Pb} /{ }^{206} \mathrm{~Pb}$ & $1 \sigma$ & ${ }^{207} \mathrm{~Pb} /{ }^{235} \mathrm{U}$ & $1 \sigma$ & ${ }^{206} \mathrm{~Pb} /{ }^{238} \mathrm{U}$ & $1 \sigma$ & ${ }^{207} \mathrm{~Pb} /{ }^{206} \mathrm{~Pb}$ & $1 \sigma$ \\
\hline YZ1803-19 & 11.27 & 108 & 252 & 0.43 & 0.2736 & 0.0154 & 0.0383 & 0.0004 & 0.0517 & 0.0029 & 246 & 12.3 & 242.5 & 2.8 & 272 & 125.9 \\
\hline YZ1803-20 & 11.11 & 130 & 239 & 0.54 & 0.2887 & 0.0127 & 0.0388 & 0.0005 & 0.0544 & 0.0025 & 258 & 10.0 & 245.1 & 2.8 & 387 & 103.7 \\
\hline YZ1803-21 & 13.67 & 180 & 297 & 0.61 & 0.2592 & 0.0122 & 0.0384 & 0.0004 & 0.0490 & 0.0023 & 234 & 9.8 & 242.6 & 2.7 & 146 & 111 \\
\hline YZ1803-22 & 11.12 & 120 & 249 & 0.48 & 0.2685 & 0.0109 & 0.0385 & 0.0004 & 0.0506 & 0.0021 & 242 & 8.7 & 243.5 & 2.7 & 220 & 101 \\
\hline YZ1803-23 & 35.6 & 219 & 446 & 0.49 & 0.5387 & 0.0198 & 0.0691 & 0.0014 & 0.0558 & 0.0016 & 438 & 13.0 & 430.8 & 8.5 & 443 & 58.3 \\
\hline YZ1803-24 & 7.63 & 61.6 & 171 & 0.36 & 0.2810 & 0.0164 & 0.0399 & 0.0006 & 0.0517 & 0.0032 & 251 & 13.0 & 252.3 & 3.6 & 272 & 141 \\
\hline YZ1803-25 & 7.63 & 69.3 & 167 & 0.41 & 0.2839 & 0.0165 & 0.0401 & 0.0006 & 0.0513 & 0.0029 & 254 & 13.1 & 253.4 & 3.6 & 254 & 136 \\
\hline YZ1803-26 & 8.80 & 94.0 & 191 & 0.49 & 0.2779 & 0.0150 & 0.0403 & 0.0006 & 0.0497 & 0.0027 & 249 & 11.9 & 254.7 & 3.4 & 183 & 124 \\
\hline \multicolumn{17}{|c|}{ YZ1804, Tuobake Granite Porphyry } \\
\hline YZ1804-01 & 8.41 & 91.7 & 186 & 0.49 & 0.2848 & 0.0172 & 0.0389 & 0.0005 & 0.0534 & 0.0033 & 254 & 13.6 & 246.0 & 2.9 & 346 & 139 \\
\hline YZ1804-02 & 11.85 & 140 & 256 & 0.55 & 0.2915 & 0.0111 & 0.0395 & 0.0004 & 0.0535 & 0.0021 & 260 & 8.7 & 250.0 & 2.6 & 350 & 90.7 \\
\hline YZ1804-03 & 10.27 & 112 & 231 & 0.49 & 0.2798 & 0.0133 & 0.0382 & 0.0004 & 0.0531 & 0.0026 & 251 & 10.6 & 241.9 & 2.7 & 345 & 111 \\
\hline YZ1804-04 & 7.46 & 68.3 & 169 & 0.40 & 0.2644 & 0.0140 & 0.0390 & 0.0005 & 0.0496 & 0.0027 & 238 & 11.3 & 246.5 & 3.2 & 176 & 130 \\
\hline YZ1804-05 & 9.28 & 102 & 202 & 0.50 & 0.2907 & 0.0161 & 0.0394 & 0.0005 & 0.0540 & 0.0032 & 259 & 12.7 & 249.0 & 3.2 & 372 & 131 \\
\hline YZ1804-06 & 8.51 & 86.8 & 190 & 0.46 & 0.2718 & 0.0156 & 0.0386 & 0.0006 & 0.0515 & 0.0031 & 244 & 12.5 & 244.3 & 3.4 & 261 & 139 \\
\hline YZ1804-07 & 6.68 & 77.4 & 153 & 0.51 & 0.2687 & 0.0133 & 0.0384 & 0.0005 & 0.0514 & 0.0027 & 242 & 10.7 & 243.1 & 3.2 & 261 & 88 \\
\hline YZ1804-09 & 8.01 & 85.1 & 178 & 0.48 & 0.2639 & 0.0130 & 0.0384 & 0.0005 & 0.0497 & 0.0025 & 238 & 10.4 & 242.8 & 3.1 & 189 & 119 \\
\hline YZ1804-10 & 26.77 & 96.5 & 662 & 0.15 & 0.2717 & 0.0093 & 0.0381 & 0.0004 & 0.0515 & 0.0019 & 244 & 7.5 & 241.1 & 2.3 & 265 & 78.7 \\
\hline YZ1804-11 & 8.49 & 88.8 & 190 & 0.47 & 0.2786 & 0.0138 & 0.0384 & 0.0005 & 0.0536 & 0.0029 & 250 & 11.0 & 242.6 & 3.2 & 354 & 122 \\
\hline YZ1804-12 & 17.75 & 228 & 376 & 0.61 & 0.2891 & 0.0101 & 0.0391 & 0.0004 & 0.0536 & 0.0020 & 258 & 8.0 & 247.4 & 2.5 & 354 & 50.9 \\
\hline YZ1804-13 & 13.98 & 178 & 302 & 0.59 & 0.2776 & 0.0102 & 0.0386 & 0.0004 & 0.0527 & 0.0021 & 249 & 8.1 & 244.0 & 2.6 & 317 & 123.1 \\
\hline YZ1804-14 & 18.24 & 103 & 421 & 0.24 & 0.2889 & 0.0111 & 0.0394 & 0.0004 & 0.0533 & 0.0021 & 258 & 8.7 & 248.8 & 2.6 & 343 & 88.9 \\
\hline YZ1804-15 & 16.51 & 110 & 382 & 0.29 & 0.2719 & 0.0102 & 0.0387 & 0.0004 & 0.0509 & 0.0019 & 244 & 8.1 & 244.5 & 2.3 & 235 & 87.0 \\
\hline YZ1804-16 & 9.75 & 106 & 215 & 0.49 & 0.2731 & 0.0123 & 0.0385 & 0.0005 & 0.0513 & 0.0023 & 245 & 9.8 & 243.4 & 3.1 & 254 & 104 \\
\hline YZ1804-17 & 10.08 & 110 & 219 & 0.50 & 0.2929 & 0.0131 & 0.0394 & 0.0005 & 0.0547 & 0.0026 & 261 & 10.3 & 248.9 & 3.2 & 467 & 103 \\
\hline YZ1804-18 & 9.37 & 96.4 & 212 & 0.45 & 0.2823 & 0.0128 & 0.0380 & 0.0005 & 0.0542 & 0.0025 & 253 & 10.1 & 240.2 & 3.0 & 389 & 105.5 \\
\hline YZ1804-19 & 13.79 & 94.4 & 319 & 0.30 & 0.2735 & 0.0101 & 0.0389 & 0.0008 & 0.0521 & 0.0021 & 245 & 8.0 & 246.0 & 5.3 & 287 & 97.2 \\
\hline YZ1804-20 & 8.73 & 81.9 & 194 & 0.42 & 0.2801 & 0.0124 & 0.0392 & 0.0005 & 0.0520 & 0.0023 & 251 & 9.9 & 248.0 & 3.0 & 283 & 97.2 \\
\hline YZ1804-21 & 27.76 & 215 & 468 & 0.46 & 0.4023 & 0.0142 & 0.0520 & 0.0009 & 0.0553 & 0.0016 & 343 & 10.3 & 326.7 & 5.5 & 433 & 60.2 \\
\hline YZ1804-22 & 9.65 & 83.0 & 223 & 0.37 & 0.2887 & 0.0147 & 0.0389 & 0.0005 & 0.0540 & 0.0028 & 258 & 11.6 & 245.8 & 2.8 & 369 & 116.7 \\
\hline YZ1804-23 & 43.8 & 517 & 921 & 0.56 & 0.2851 & 0.0081 & 0.0408 & 0.0004 & 0.0506 & 0.0015 & 255 & 6.4 & 257.8 & 2.4 & 220 & 66.7 \\
\hline YZ1804-24 & 8.23 & 79.4 & 186 & 0.43 & 0.2971 & 0.0133 & 0.0386 & 0.0005 & 0.0563 & 0.0027 & 264 & 10.4 & 244.0 & 3.4 & 465 & 101 \\
\hline YZ1804-25 & 9.04 & 107 & 203 & 0.53 & 0.2644 & 0.0160 & 0.0385 & 0.0005 & 0.0496 & 0.0029 & 238 & 12.9 & 243.6 & 3.0 & 176 & 133 \\
\hline
\end{tabular}




\subsection{Zircon Hf Isotopic Compositions}

Zircon Lu-Hf isotopic data of the above four granite samples are shown in Table 4. A total of thirty-one zircons from the Zengudi granite porphyries and thirty-one zircons from the Tuobake granite porphyries were analyzed for ${ }^{176} \mathrm{Hf} /{ }^{177} \mathrm{Hf}$ isotopic ratios. The ${ }^{176} \mathrm{Lu} /{ }^{177} \mathrm{Hf}$ ratios of all the analyzed zircons are less than 0.002 , indicating that there is no radiogenic $\mathrm{Hf}$ accumulation after zircon formation [56]. Thus, the results of ${ }^{176} \mathrm{Hf} /{ }^{177} \mathrm{Hf}$ ratios can represent the $\mathrm{Hf}$ isotopic composition of the magmatic system.

Zircons of the Zengudi granite porphyries show variable Hf isotopic compositions with ${ }^{176} \mathrm{Hf} /{ }^{177} \mathrm{Hf}$ ratios of $0.282256-0.282543$. The calculated $\varepsilon_{\mathrm{Hf}}(\mathrm{t})$ values range between -12.94 and -2.63 , which correspond to the crustal model age $\left(\mathrm{T}_{\mathrm{DM}}{ }^{\mathrm{C}}\right)$ of $1080-2095 \mathrm{Ma}$.

Zircons of the Tuobake granite porphyries have more variable ${ }^{176} \mathrm{Hf} /{ }^{177} \mathrm{Hf}$ ratios ranging from 0.282222 to 0.282440 . The calculated $\varepsilon_{\mathrm{Hf}}(\mathrm{t})$ values vary from -14.06 to -6.55 , which correspond to the crustal model age $\left(\mathrm{T}_{\mathrm{DM}}{ }^{\mathrm{C}}\right)$ of $1689-2164 \mathrm{Ma}$.

Table 4. LA-MC-ICPMS zircon Hf isotopic data of zircon grains from the Zengudi and Tuobake granite porphyries.

\begin{tabular}{|c|c|c|c|c|c|c|c|c|c|c|c|}
\hline Spot & ${ }^{176} \mathrm{Yb} /{ }^{177} \mathrm{Hf}$ & ${ }^{176} \mathrm{Lu} /{ }^{177} \mathrm{Hf}$ & ${ }^{176} \mathrm{Hf} /{ }^{177} \mathrm{Hf}$ & $2 \sigma$ & Age(Ma) & $\left({ }^{176} \mathrm{Hf} /{ }^{177} \mathrm{Hf}\right)_{\mathrm{i}}$ & $\varepsilon_{\mathrm{Hf}}(0)$ & $\varepsilon_{H f}(t)$ & $\mathbf{T}_{\mathrm{DM}}$ & $\mathbf{T}_{\mathrm{DMc}}$ & $f_{\mathrm{Lu} / \mathrm{Hf}}$ \\
\hline \multicolumn{12}{|c|}{ SC1801, Zengudi Granite Porphyry } \\
\hline 1 & 0.052434 & 0.001550 & 0.282458 & 0.000009 & 254 & 0.282450 & -11.12 & -5.81 & 1140 & 1649 & -0.95 \\
\hline 2 & 0.015455 & 0.000515 & 0.282427 & 0.000008 & 254 & 0.282425 & -12.20 & -6.70 & 1151 & 1706 & -0.98 \\
\hline 3 & 0.024921 & 0.000829 & 0.282494 & 0.000008 & 254 & 0.282490 & -9.82 & -4.40 & 1067 & 1559 & -0.98 \\
\hline 4 & 0.021883 & 0.000770 & 0.282533 & 0.000008 & 254 & 0.282530 & -8.45 & -2.98 & 1011 & 1471 & -0.98 \\
\hline 5 & 0.035953 & 0.001084 & 0.282409 & 0.000008 & 254 & 0.282404 & -12.82 & -7.44 & 1193 & 1752 & -0.97 \\
\hline 6 & 0.025831 & 0.000866 & 0.282493 & 0.000008 & 254 & 0.282489 & -9.88 & -4.43 & 1070 & 1563 & -0.97 \\
\hline 7 & 0.020342 & 0.000666 & 0.282437 & 0.000008 & 254 & 0.282434 & -11.85 & -6.38 & 1142 & 1686 & -0.98 \\
\hline 8 & 0.016995 & 0.000576 & 0.282483 & 0.000009 & 254 & 0.282480 & -10.23 & -4.75 & 1076 & 1583 & -0.98 \\
\hline 9 & 0.017185 & 0.000605 & 0.282543 & 0.000009 & 254 & 0.282540 & -8.09 & -2.63 & 992 & 1446 & -0.98 \\
\hline 10 & 0.034599 & 0.001078 & 0.282469 & 0.000008 & 254 & 0.282464 & -10.71 & -5.32 & 1109 & 1618 & -0.97 \\
\hline 11 & 0.034650 & 0.001123 & 0.282493 & 0.000008 & 254 & 0.282487 & -9.88 & -4.50 & 1077 & 1565 & -0.97 \\
\hline 12 & 0.022910 & 0.000765 & 0.282503 & 0.000007 & 254 & 0.282500 & -9.50 & -4.04 & 1052 & 1538 & -0.98 \\
\hline 13 & 0.039795 & 0.001268 & 0.282488 & 0.000008 & 254 & 0.282482 & -10.04 & -4.68 & 1088 & 1578 & -0.96 \\
\hline 14 & 0.022477 & 0.000753 & 0.282508 & 0.000007 & 254 & 0.282504 & -9.34 & -3.90 & 1045 & 1527 & -0.98 \\
\hline \multicolumn{12}{|c|}{ SC1802, Zengudi Granite Porphyry } \\
\hline 1 & 0.051045 & 0.001464 & 0.282415 & 0.000010 & 246 & 0.282408 & -12.63 & -7.46 & 1198 & 1748 & -0.96 \\
\hline 2 & 0.041120 & 0.001160 & 0.282363 & 0.000008 & 246 & 0.282357 & -14.48 & -9.26 & 1261 & 1861 & -0.97 \\
\hline 3 & 0.049009 & 0.001353 & 0.282371 & 0.000007 & 246 & 0.282365 & -14.17 & -8.98 & 1255 & 1843 & -0.96 \\
\hline 4 & 0.034964 & 0.000950 & 0.282324 & 0.000008 & 246 & 0.282319 & -15.85 & -10.61 & 1309 & 1947 & -0.97 \\
\hline 5 & 0.050138 & 0.001380 & 0.282325 & 0.000010 & 246 & 0.282319 & -15.81 & -10.61 & 1322 & 1948 & -0.96 \\
\hline 6 & 0.042443 & 0.001181 & 0.282376 & 0.000008 & 246 & 0.282370 & -14.02 & -8.80 & 1244 & 1833 & -0.96 \\
\hline 7 & 0.022381 & 0.000578 & 0.282256 & 0.000010 & 246 & 0.282253 & -18.25 & -12.94 & 1390 & 2095 & -0.98 \\
\hline 8 & 0.043054 & 0.001270 & 0.282261 & 0.000009 & 246 & 0.282256 & -18.06 & -12.84 & 1407 & 2088 & -0.96 \\
\hline 9 & 0.065127 & 0.001787 & 0.282328 & 0.000009 & 246 & 0.282319 & -15.72 & -10.61 & 1333 & 1946 & -0.95 \\
\hline 10 & 0.043391 & 0.001239 & 0.282355 & 0.000009 & 246 & 0.282350 & -14.73 & -9.51 & 1274 & 1878 & -0.96 \\
\hline 11 & 0.033480 & 0.000914 & 0.282276 & 0.000008 & 246 & 0.282272 & -17.53 & -12.27 & 1374 & 2053 & -0.97 \\
\hline 12 & 0.041500 & 0.001133 & 0.282359 & 0.000007 & 246 & 0.282354 & -14.59 & -9.37 & 1265 & 1869 & -0.97 \\
\hline 13 & 0.047639 & 0.001299 & 0.282390 & 0.000008 & 246 & 0.282384 & -13.50 & -8.31 & 1227 & 1801 & -0.96 \\
\hline 14 & 0.051748 & 0.001448 & 0.282353 & 0.000008 & 246 & 0.282346 & -14.83 & -9.65 & 1285 & 1886 & -0.96 \\
\hline 15 & 0.041658 & 0.001197 & 0.282395 & 0.000009 & 246 & 0.282390 & -13.32 & -8.10 & 1216 & 1788 & -0.96 \\
\hline 16 & 0.039886 & 0.001110 & 0.282370 & 0.000008 & 246 & 0.282365 & -14.21 & -8.98 & 1249 & 1844 & -0.97 \\
\hline \multicolumn{12}{|c|}{ YZ1803, Tuobake Granite Porphyry } \\
\hline 1 & 0.056035 & 0.001588 & 0.282229 & 0.000009 & 246 & 0.282222 & -19.19 & -14.06 & 1465 & 2164 & -0.95 \\
\hline 2 & 0.045067 & 0.001288 & 0.282418 & 0.000008 & 246 & 0.282412 & -12.51 & -7.33 & 1187 & 1738 & -0.96 \\
\hline 3 & 0.033968 & 0.000948 & 0.282435 & 0.000007 & 246 & 0.282430 & -11.93 & -6.70 & 1154 & 1699 & -0.97 \\
\hline 4 & 0.055386 & 0.001535 & 0.282325 & 0.000007 & 246 & 0.282318 & -15.80 & -10.66 & 1327 & 1949 & -0.95 \\
\hline 5 & 0.055885 & 0.001572 & 0.282409 & 0.000007 & 246 & 0.282402 & -12.83 & -7.69 & 1209 & 1762 & -0.95 \\
\hline 6 & 0.047681 & 0.001319 & 0.282295 & 0.000007 & 246 & 0.282289 & -16.88 & -11.68 & 1363 & 2016 & -0.96 \\
\hline 7 & 0.040449 & 0.001194 & 0.282027 & 0.000017 & 246 & 0.282021 & -26.36 & -11.17 & 1233 & 1910 & -0.96 \\
\hline 8 & 0.042754 & 0.001176 & 0.282398 & 0.000009 & 246 & 0.282393 & -13.22 & -8.00 & 1212 & 1783 & -0.96 \\
\hline 9 & 0.047543 & 0.001350 & 0.282298 & 0.000008 & 246 & 0.282292 & -16.75 & -11.58 & 1358 & 2007 & -0.96 \\
\hline 10 & 0.036930 & 0.000811 & 0.282442 & 0.000014 & 246 & 0.282138 & -10.96 & -11.32 & 1244 & 1756 & -0.98 \\
\hline 11 & 0.030327 & 0.000870 & 0.282403 & 0.000008 & 246 & 0.282399 & -13.06 & -7.79 & 1196 & 1770 & -0.97 \\
\hline 12 & 0.039229 & 0.001104 & 0.282429 & 0.000008 & 246 & 0.282424 & -12.11 & -6.91 & 1166 & 1712 & -0.97 \\
\hline 13 & 0.052511 & 0.001464 & 0.282416 & 0.000008 & 246 & 0.282409 & -12.60 & -7.44 & 1196 & 1746 & -0.96 \\
\hline 14 & 0.059767 & 0.001652 & 0.282440 & 0.000008 & 246 & 0.282432 & -11.75 & -6.62 & 1168 & 1694 & -0.95 \\
\hline
\end{tabular}


Table 4. Cont.

\begin{tabular}{|c|c|c|c|c|c|c|c|c|c|c|c|}
\hline Spot & ${ }^{176} \mathrm{Yb} /{ }^{177} \mathrm{Hf}$ & ${ }^{176} \mathrm{Lu} /{ }^{177} \mathrm{Hf}$ & ${ }^{176} \mathrm{Hf} /{ }^{177} \mathrm{Hf}$ & $2 \sigma$ & Age(Ma) & $\left({ }^{176} \mathrm{Hf} /{ }^{177} \mathrm{Hf}\right)_{\mathrm{i}}$ & $\varepsilon_{\mathrm{Hf}}(0)$ & $\varepsilon_{H f}(t)$ & $\mathbf{T}_{\mathrm{DM}}$ & $\mathrm{T}_{\mathrm{DMc}}$ & $f_{\mathrm{Lu} / \mathrm{Hf}}$ \\
\hline \multicolumn{12}{|c|}{ YZ1804, Tuobake Granite Porphyry } \\
\hline 1 & 0.056426 & 0.001536 & 0.282440 & 0.000009 & 246 & 0.282433 & -11.75 & -6.59 & 1164 & 1692 & -0.95 \\
\hline 2 & 0.048878 & 0.001315 & 0.282422 & 0.000009 & 246 & 0.282416 & -12.37 & -7.19 & 1182 & 1729 & -0.96 \\
\hline 3 & 0.060706 & 0.001661 & 0.282279 & 0.000008 & 246 & 0.282272 & -17.43 & -12.29 & 1397 & 2053 & -0.95 \\
\hline 4 & 0.051981 & 0.001418 & 0.282423 & 0.000007 & 246 & 0.282417 & -12.33 & -7.16 & 1184 & 1728 & -0.96 \\
\hline 5 & 0.046330 & 0.001293 & 0.282440 & 0.000014 & 246 & 0.282434 & -11.73 & -6.55 & 1156 & 1689 & -0.96 \\
\hline 6 & 0.047305 & 0.001318 & 0.282353 & 0.000011 & 246 & 0.282347 & -14.81 & -9.63 & 1280 & 1885 & -0.96 \\
\hline 7 & 0.047661 & 0.001313 & 0.282428 & 0.000009 & 246 & 0.282422 & -12.17 & -6.98 & 1174 & 1717 & -0.96 \\
\hline 8 & 0.049598 & 0.001395 & 0.282424 & 0.000009 & 246 & 0.282418 & -12.30 & -7.12 & 1182 & 1726 & -0.96 \\
\hline 9 & 0.045268 & 0.001254 & 0.282413 & 0.000008 & 246 & 0.282408 & -12.69 & -7.47 & 1193 & 1749 & -0.96 \\
\hline 10 & 0.053964 & 0.001442 & 0.282263 & 0.000007 & 246 & 0.282257 & -17.99 & -12.82 & 1411 & 2086 & -0.96 \\
\hline 11 & 0.051494 & 0.001401 & 0.282416 & 0.000007 & 246 & 0.282409 & -12.60 & -7.44 & 1194 & 1745 & -0.96 \\
\hline 12 & 0.032045 & 0.000872 & 0.282409 & 0.000009 & 246 & 0.282405 & -12.82 & -7.58 & 1187 & 1755 & -0.97 \\
\hline 14 & 0.050771 & 0.001369 & 0.282369 & 0.000008 & 246 & 0.282362 & -14.27 & -9.10 & 1260 & 1851 & -0.96 \\
\hline 15 & 0.102921 & 0.002808 & 0.282292 & 0.000008 & 246 & 0.282279 & -16.98 & -12.04 & 1423 & 2036 & -0.92 \\
\hline 16 & 0.068002 & 0.001850 & 0.282397 & 0.000010 & 246 & 0.282389 & -13.26 & -8.15 & 1235 & 1791 & -0.94 \\
\hline 17 & 0.064255 & 0.001732 & 0.282429 & 0.000008 & 246 & 0.282421 & -12.12 & -7.01 & 1186 & 1719 & -0.95 \\
\hline
\end{tabular}

\section{Discussion}

\subsection{Genetic Type and Magma Origin of the Granite Porphyries}

Granitic rocks have been commonly classified into I-, M-, S-, and A- type granites [57-60], largely based on their petrographic and geochemical characteristics, and the nature of magma sources. The studied granite porphyries are geochemically distinct from A- and M-type granites, which are both characterized by low $\mathrm{K}_{2} \mathrm{O}(<1 \mathrm{wt} . \%)$ and high alkali index ( $\mathrm{AI}>0.85)$, respectively [61]. In this study, the Zengudi granite porphyries exhibit high contents of $\mathrm{SiO}_{2}(68.04-87.61 \%)$ and $\mathrm{Al}_{2} \mathrm{O}_{3}(5.55-18.33 \%)$, low contents of $\mathrm{P}_{2} \mathrm{O}_{5}$ $(0.043-0.16 \%)$ and HFSE, low alkali index $(\mathrm{AI}=0.31-0.69)$ and high $\mathrm{A} / \mathrm{CNK}$ ratios of 1.07-1.86 (Table 1). In addition, these granitic rocks display typical ratios of $\mathrm{Fe}_{2} \mathrm{O}_{3}{ }^{\mathrm{T}} / \mathrm{MgO}$ (2.24-3.29) and 10,000 $\times \mathrm{Ga} / \mathrm{Al}$ of 2.19-2.66, $\mathrm{Zr}$ (12-300 ppm) and $\mathrm{Nb}$ (6.39-15.6 ppm) contents. Thus, these geochemical features rule out the A-type affinity for the Zengudi granite porphyries $[2,62,63]$. Moreover, the contents of Th and $Y$ do not vary with increasing $\mathrm{Rb}$ content (Figure $7 \mathrm{a}, \mathrm{b}$ ), and the $\mathrm{Sr} / \mathrm{Ba}$ ratios range from 0.10 to 0.24 . Overall, these characteristics indicate that the Zengudi granite porphyries are S-type granites.

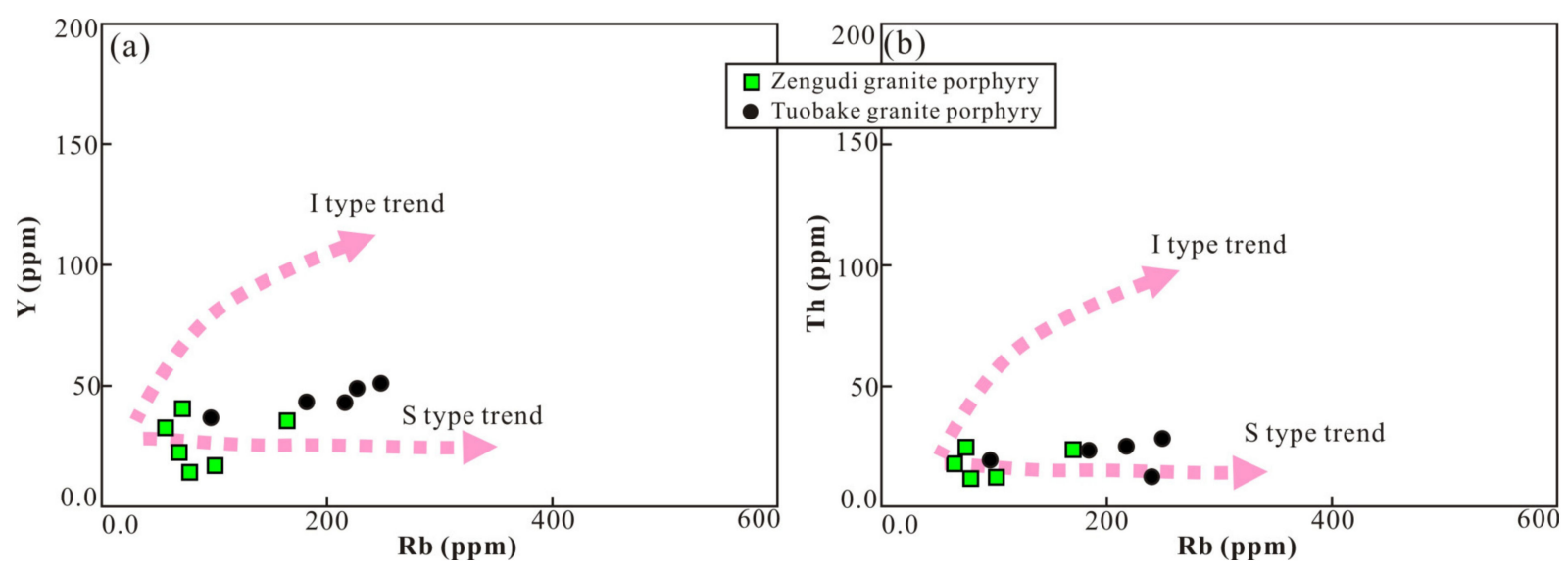

Figure 7. Classification diagrams of the Zengudi and Tuobake granite porphyries. (a) Rb versus Y and (b) Rb versus Th [57].

The Tuobake granite porphyries have relatively high $\mathrm{SiO}_{2}(71.07-77.75 \%)$ and $\mathrm{Al}_{2} \mathrm{O}_{3}$ (9.21-13.98\%), and low $\mathrm{P}_{2} \mathrm{O}_{5}(9.21-13.98 \%)$ contents (Table 1). Except sample YZ-H3, their A/CNK ratios are mostly higher than 1.10 (Table 1). This suggests that the Tuobake granite porphyries are peraluminous granites. These granites also display low AI values (0.51-0.68) and low contents of HFSE such as $\mathrm{Nb}(9.57-15.0 \mathrm{ppm})$ and $\mathrm{Zr}$ (181-289 ppm), with typical 
ratios of $10,000 \times \mathrm{Ga} / \mathrm{Al}(1.97-2.81)$ and $\mathrm{Fe}_{2} \mathrm{O}_{3}{ }^{\mathrm{T}} / \mathrm{MgO}$ (3.90-5.86). Moreover, the Th contents of these granites do not increase with increasing $\mathrm{Rb}$ content, while their $\mathrm{Y}$ contents exhibit a slight positive correlation with increasing $\mathrm{Rb}$ (Figure 7a,b). Hence, these features suggest that the Tuobake granite porphyries are S-type granites.

Most peraluminous granites are formed by partial melting of crustal rocks [64-67] or metamorphosed sediments such as (1) metamorphosed mudstones and sandstones [66], (2) metamorphosed felsic igneous rocks (orthogneiss) [68], and (3) metamorphosed basaltic igneous rocks $[69,70]$. Previous studies have documented that the granitic magma originated from the orthogneiss or sandstone source exhibits high $\mathrm{CaO} / \mathrm{Na}_{2} \mathrm{O}$ ratios and low $\mathrm{Al}_{2} \mathrm{O}_{3} / \mathrm{TiO}_{2}$ ratios $[69,71]$. The studied Zengudi and Tuobake granite samples show high $\mathrm{CaO} / \mathrm{Na}_{2} \mathrm{O}$ ratios (0.08-11.97) and low $\mathrm{Al}_{2} \mathrm{O}_{3} / \mathrm{TiO}_{2}$ ratios (17.09-40.32). This indicates that partial melting of orthogneiss or sandstone in the source region may have produced the original magma for these granitic rocks. In the $\mathrm{Na}_{2} \mathrm{O} / \mathrm{CaO}$ versus $\mathrm{Al}_{2} \mathrm{O}_{3} / \mathrm{TiO}_{2}$ diagram (Figure 8a), most granite samples (except samples SC-H2 and SC-H3) plot in the field of psammitolite source.
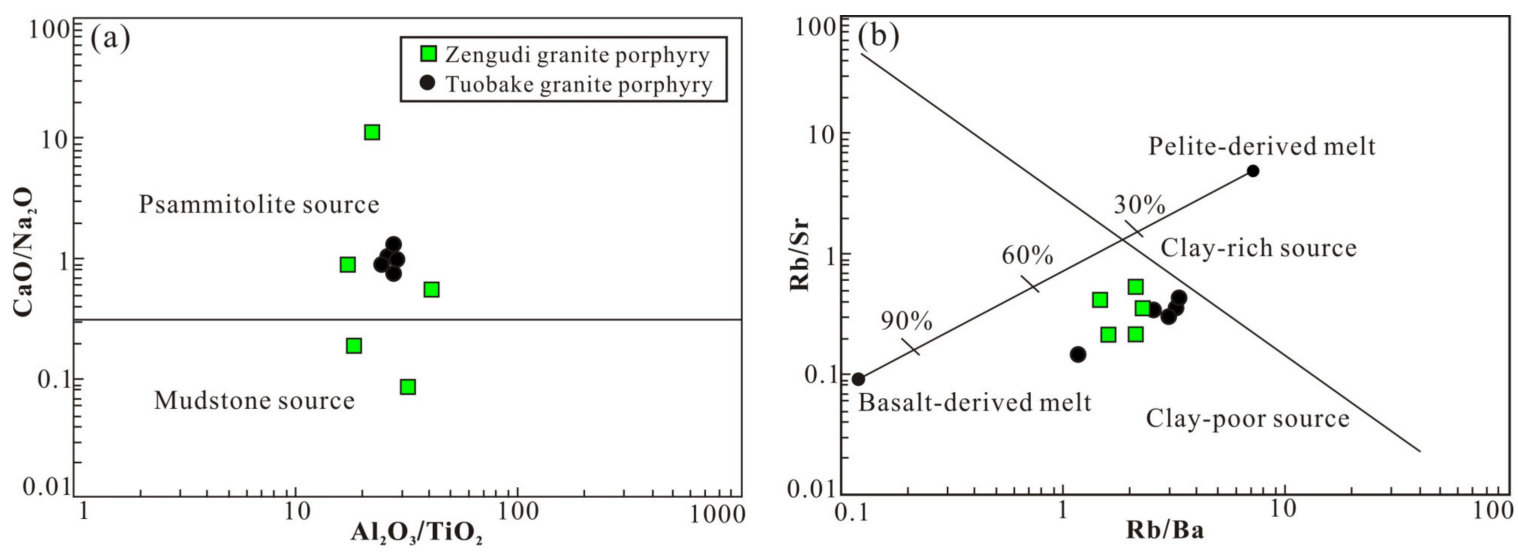

Figure 8. Magma source discrimination diagrams of the Zengudi and Tuobake granite porphyries. (a) $\mathrm{Na}_{2} \mathrm{O} / \mathrm{CaO}$ versus $\mathrm{Al}_{2} \mathrm{O}_{3} / \mathrm{TiO}_{2}$ and (b) $\mathrm{Rb} / \mathrm{Sr}$ vs. $\mathrm{Rb} / \mathrm{Ba}[66]$.

The $\mathrm{w}(\mathrm{CaO}) / \mathrm{w}\left(\mathrm{Na}_{2} \mathrm{O}\right)$ ratios are usually used as a powerful index for distinguishing the source of granitic magma $[58,65]$. The granites originated from a clay-rich source are characterized by $\mathrm{w}(\mathrm{CaO}) / \mathrm{w}\left(\mathrm{Na}_{2} \mathrm{O}\right)$ ratio $<0.3$, whereas $\mathrm{w}(\mathrm{CaO}) / \mathrm{w}\left(\mathrm{Na}_{2} \mathrm{O}\right)$ ratio $>0.3$ reflects granites originated from a plagioclase-rich and clay-poor clastic source [72]. In this study, the $\mathrm{w}(\mathrm{CaO}) / \mathrm{w}\left(\mathrm{Na}_{2} \mathrm{O}\right)$ ratios of the Zengudi and Tuobake granite porphyries are mostly $>0.3(0.08-11.97$ and $0.8-1.20$, respectively), indicating that their granitic magmas were generated from a plagioclase-rich and clay-poor clastic source. In the $\mathrm{Rb} / \mathrm{Sr}$ versus $\mathrm{Rb} / \mathrm{Ba}$ diagram (Figure $8 \mathrm{~b}$ ), all samples plot in the field of clay-poor source and show a close affinity to pelite-derived melt. This further corroborates that the Zengudi and Tuobake granite porphyries were formed by the partial melting of psammitolite.

The Nd isotopic compositions of granitic rocks and their Hf isotopes of zircons can further reflect their magma sources $[9,54,66,73]$. The studied Zengudi and Tuobake granite porphyries show a narrow negative range of $\varepsilon_{\mathrm{Nd}}(\mathrm{t})$ values $(-14.49$ to -9.35$)$ (Table 2). The corresponding $\mathrm{Nd}$ model ages $\left(\mathrm{T}_{\mathrm{DM}}{ }^{\mathrm{C}}\right)$ vary from 1.78 to $2.46 \mathrm{Ga}$, suggesting that their magmas were derived from remelting of crustal source. The zircons from the Zengudi and Tuobake granite porphyries also exhibit negative $\varepsilon_{\mathrm{Hf}}(\mathrm{t})$ values (mostly -14 to -3 ) (Table 2), and have crustal model ages $\left(\mathrm{T}_{\mathrm{DM}}{ }^{\mathrm{C}}\right)$ of 1.08-2.2 Ga. This indicates that the magma producing the studied granitic rocks originated from an old crustal source. On the $\varepsilon_{N d}(t)$ versus $\varepsilon_{H f}(t)$ diagram (Figure $9 a$ ), all the granite samples plot in the field of lower continental crust and global sediments, which reflects an old crustal source for the magmas. Moreover, on the $\varepsilon_{\mathrm{Nd}}(\mathrm{t})$ versus $\left({ }^{87} \mathrm{Sr} /{ }^{86} \mathrm{Sr}\right)_{\mathrm{i}}$ diagram (Figure $9 \mathrm{~b}$ ), two samples of the Zengudi granite porphyries show a close affinity with the S-type granitoids from the Lancangjiang fault belt (LFB), while four samples of the Tuobake granite porphyries plot 
in the field of Lancangjiang granitoids formed from the Indochina block (Simao Terrane). Thus, we infer that the Lancangjiang Paleo-Tethys suture zone is located at the west of this study area. The geochemical analyses of the studied granites show that these granite porphyries are strongly enriched in LILE (e.g., $\mathrm{Rb}$, Th, $\mathrm{K}$ and $\mathrm{U}$ ) but depleted in HFSE (e.g., $\mathrm{Ta}, \mathrm{Nb}$, and Ti) (Figure $4 \mathrm{~b}$ ), with high $\left({ }^{87} \mathrm{Sr} /{ }^{86} \mathrm{Sr}\right)_{\mathrm{i}}$ and low $\varepsilon_{\mathrm{Nd}}(\mathrm{t})$ values. Overall, these granites were sourced from magmas of continental crust origin.
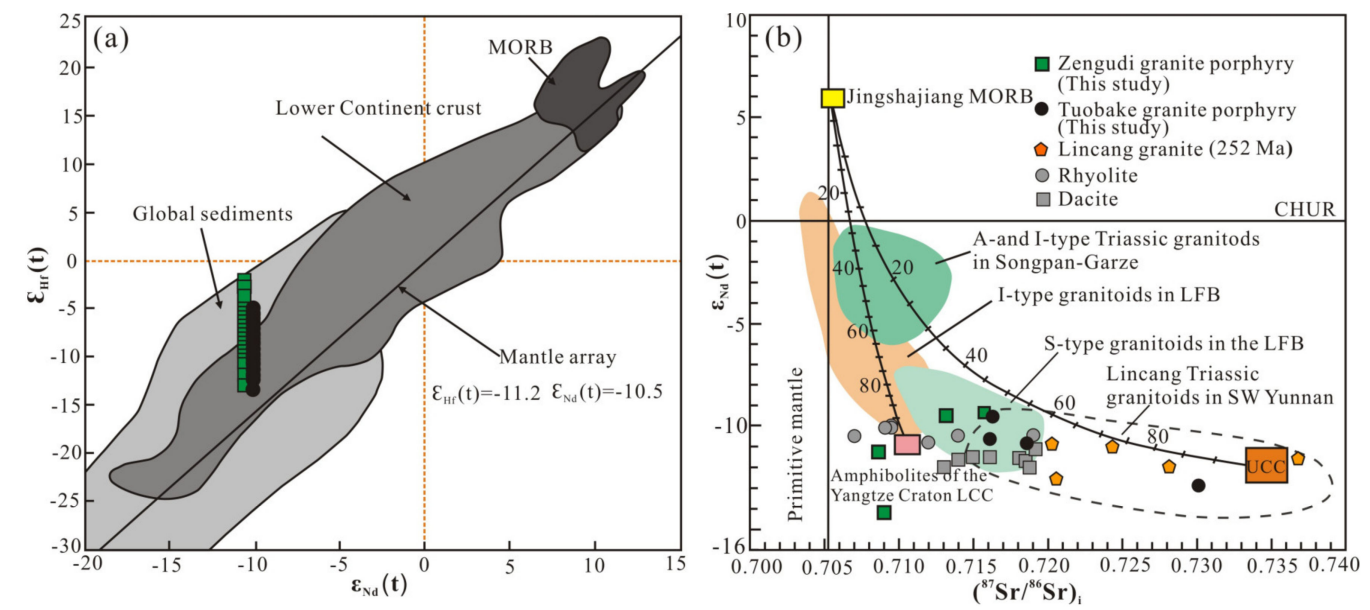

Figure 9. (a) Plots of $\varepsilon_{\mathrm{Hf}}(\mathrm{t})$ versus $\varepsilon_{\mathrm{Nd}}(\mathrm{t})$ and $(\mathbf{b}) \varepsilon_{\mathrm{Nd}}(\mathrm{t})$ versus $\left({ }^{87} \mathrm{Sr} /{ }^{86} \mathrm{Sr}\right)_{\mathrm{i}}$ of the Zengudi and Tuobake granite porphyries. The initial isotopic ratios of the granite porphyries were corrected to $254 \mathrm{Ma}$ and $246 \mathrm{Ma}$, respectively [16,17,22]. The Jinshajiang MORB is from [74]; Gangdese I-type granite is from [75]; I-type and S-type granites in Lancangjiang fault belt (LFB) is from [76]; and the Lancangjiang granite is from [15]. The Jinshajiang MORB represents mantle-derived magmas: $\varepsilon \mathrm{Nd}(\mathrm{t})=6.1, \mathrm{Nd}=7.2 \mathrm{ppm},\left({ }^{87} \mathrm{Sr} /{ }^{86} \mathrm{Sr}\right)_{\mathrm{i}}=0.7054, \mathrm{Sr}=260 \mathrm{ppm}$. The rocks from the Xiongsong Group [77] were considered to be formed by the melting of the upper continental crust (UCC): $\varepsilon N d(t)=-12.2, \mathrm{Nd}=27.6 \mathrm{ppm}$, $\left({ }^{87} \mathrm{Sr} /{ }^{86} \mathrm{Sr}\right)_{\mathrm{i}}=0.7357, \mathrm{Sr}=168 \mathrm{ppm}$. The amphibolites of the Yangtze Craton LCC (lower continental crust $)(\varepsilon N d(t)=-10$, $\left.\mathrm{Nd}=12 \mathrm{ppm},\left({ }^{87} \mathrm{Sr} /{ }^{86} \mathrm{Sr}\right)_{\mathrm{i}}=0.710, \mathrm{Sr}=260 \mathrm{ppm}\right)$ were estimated by [78].

In the $\varepsilon_{\mathrm{Hf}}(\mathrm{t})$ versus $\mathrm{U}-\mathrm{Pb}$ age diagram (Figure 10), most granite samples plot along the crustal evolution line. Furthermore, the $\mathrm{Nb} / \mathrm{Ta}$ (11.44-13.48) and $\mathrm{Zr} / \mathrm{Hf}$ (37.42-38.55) ratios of the studied granite porphyries are comparable to those of the continental crust $[79,80]$. Combined with their significant depletion in Ba and Sr (Figure 4b), all these characteristics suggest that the granitic magmas originated from the melting of Paleoproterozoic to Mesoproterozoic crustal materials.
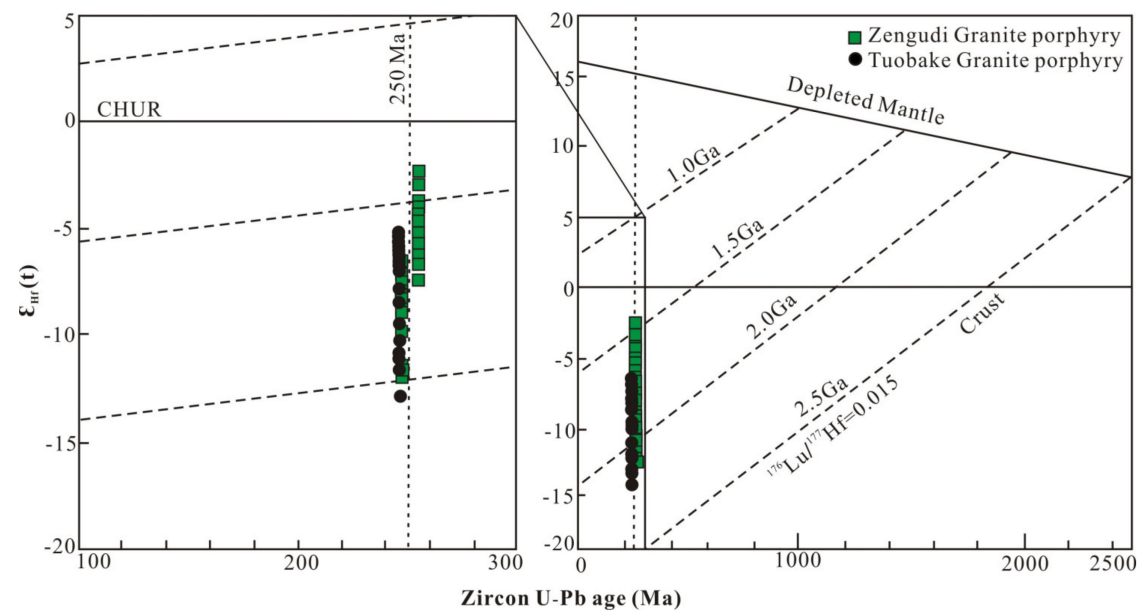

Figure 10. $\mathrm{U}-\mathrm{Pb}$ ages versus $\varepsilon_{\mathrm{Hf}}(\mathrm{t})$ for zircons from the Zengudi and Tuobake granite porphyries in the Yezhi area. The values used for constructing the depleted mantle and crustal evolution reference lines were taken from [81]. 


\subsection{Magma Evolutionary Processes of the Granite Porphyries}

The Zengudi and Tuobake granite porphyries exhibit a wide range of $\mathrm{SiO}_{2}$ concentrations (69.68-87.61\%). Moreover, $\mathrm{SiO}_{2}$ shows a clear negative correlation with major oxides (e.g., $\mathrm{TiO}_{2}, \mathrm{P}_{2} \mathrm{O}_{5}, \mathrm{CaO}, \mathrm{Al}_{2} \mathrm{O}_{3}, \mathrm{TFe}_{2} \mathrm{O}_{3}, \mathrm{MgO}$ ) and trace elements (e.g., $\mathrm{Sc}, \mathrm{Cr}$, $\mathrm{Sr}$, $\mathrm{Ni}$, and $\mathrm{Zr}$ (Figure 11). This suggests a strong fractionation crystallization during magma evolution for the studied granites. On the La/Sm versus La diagram (Figure 12f), it is noticeable that the fractional crystallization is the main factor controlling the magma evolution. The conspicuous negative anomalies of Eu, low $\sum$ HREE (8.65-30.7 ppm), and flat HREE patterns (Figure 4a) suggest that there were residual minerals (e.g., plagioclase and amphibole) in the magma source. The negative anomalies of Ba, Sr (Figure $4 \mathrm{~b}$ ) and $\mathrm{Eu}$ (Figure 4a) imply the fractional crystallization of amphibole and plagioclase during magmatic evolution, respectively. On the other hand, the negative correlation between Sc, $\mathrm{Cr}, \mathrm{Sr}, \mathrm{Ni}$, and $\mathrm{Zr}$ versus $\mathrm{SiO}_{2}$ (Figure 12) further confirms that the granitic magmas have undergone hornblende and plagioclase fractional crystallization during their magmatic evolutionary processes [82].
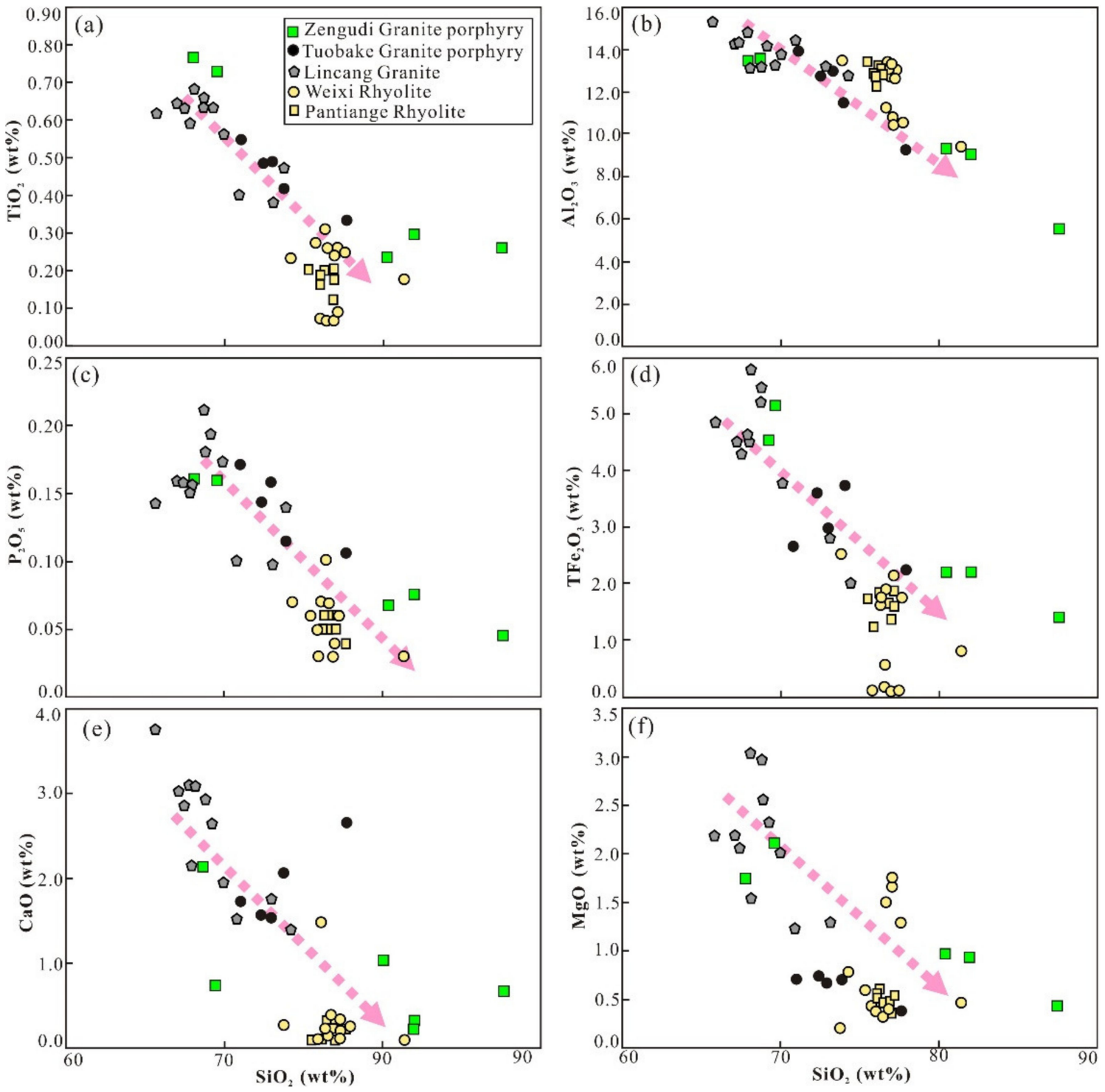

Figure 11. Harker diagrams of major elements of the Zengudi and Tuobake granite porphyries. (a) $\mathrm{SiO}_{2} \mathrm{versus}^{\mathrm{TiO}} \mathrm{O}_{2}$ (b) $\mathrm{SiO}_{2}$ versus $\mathrm{AI}_{2} \mathrm{O}_{3}$, (c) $\mathrm{SiO}_{2}$ versus $\mathrm{P}_{2} \mathrm{O}_{5}$, (d) $\mathrm{SiO}_{2}$ versus $\mathrm{TFe}_{2} \mathrm{O}_{3}$, (e) $\mathrm{SiO}_{2}$ versus $\mathrm{CaO}$, and (f) $\mathrm{SiO}_{2}$ versus $\mathrm{MgO}_{\text {. }}$ 

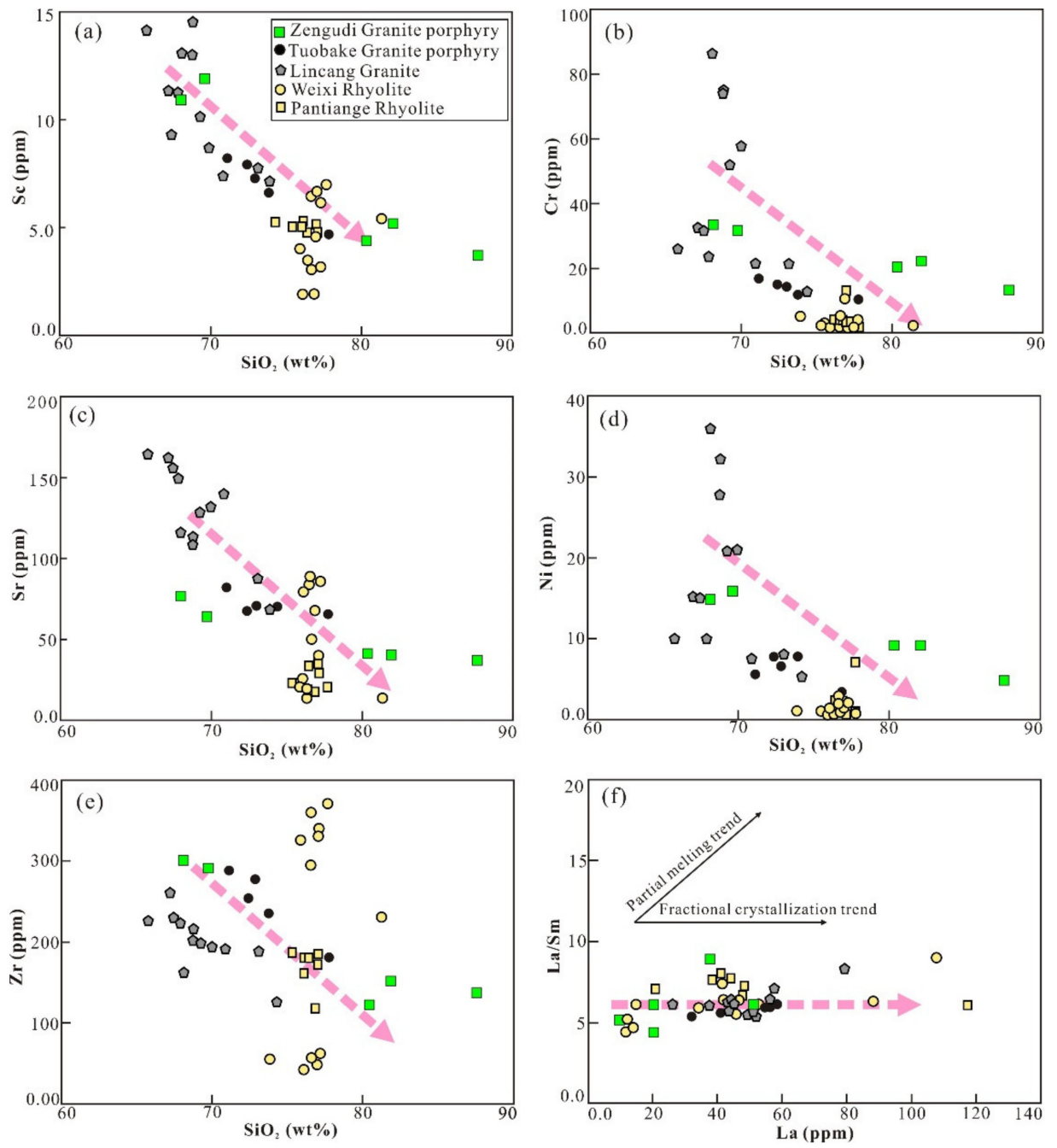

Figure 12. Harker diagrams of trace elements for the Zengudi and Tuobake granite porphyries. (a) $\mathrm{SiO}_{2}$ versus $\mathrm{Sc}$, (b) $\mathrm{SiO}_{2}$ versus $\mathrm{Cr}$, (c) $\mathrm{SiO}_{2}$ versus $\mathrm{Sr}$, (d) $\mathrm{SiO}_{2}$ versus $\mathrm{Cr}$, (e) $\mathrm{SiO}_{2}$ versus $\mathrm{Zr}$, and (f) $\mathrm{La} / \mathrm{Sm}$ versus $\mathrm{La}$.

The $\mathrm{Y} / \mathrm{Yb}$ and $(\mathrm{Ho} / \mathrm{Yb})_{\mathrm{N}}$ ratios are commonly applied to distinguish the residual phases in the magma source [83]. If $\mathrm{Y} / \mathrm{Yb}>10$ and $\mathrm{Ho}_{\mathrm{N}} / \mathrm{Yb}_{\mathrm{N}}>1.2$, garnet is the main residue mineral, whereas $\mathrm{Y} / \mathrm{Yb} \approx 10$ and $(\mathrm{Ho} / \mathrm{Yb})_{\mathrm{N}}=1$ indicate that hornblende is the residue mineral [83]. In this study, the $\mathrm{Y} / \mathrm{Yb}$ ratios range between 9.66 and 12.21 (average $=11.08)$, and the $(\mathrm{Ho} / \mathrm{Yb})_{\mathrm{N}}$ ratios vary from 0.97 to 1.21 (average $=1.12$ ). Thus, we infer that the hornblende is the major residue mineral in the magma source for the Zengudi and Tuobake granite porphyries.

In summary, the Zengudi and Tuobake granite porphyries have likely undergone strong fractionation crystallization processes of plagioclase and amphibole (e.g., hornblende).

\subsection{Tectonic Setting of the Granite Porphyries and Its Implication for the Closure of the Paleo-Tethys Ocean}

The regional tectonic evolution of the Lancangjiang area (Figure $1 \mathrm{~b}$ ) has been debated for a decade $[6,14-17,36]$. Two possible subduction events related to the formation of the Jiangda-Weixi volcanic arc have been proposed: (1) westward subduction of the Jinshajiang Ocean $[19,20,84]$, and (2) eastward subduction of the Lancangjiang Ocean $[17,21,24,85]$. Additionally, several tectonic setting models for the Triassic granitoids and bimodal volcanic rocks in the Lancangjiang area were postulated such as: (1) the Paleo-Tethys Ocean experienced three stages of tectonic evolution including a pre-collisional (ca. 255-250 Ma), 
syn-collisional (249-237 Ma), and post-collisional (236-212 Ma) setting [16]; (2) the Early Triassic and Mid Triassic magmatism were generated in volcanic arc and post-collisional extension settings, respectively [14,15]; (3) the Lincang granites (southern extension of the Baimaxueshan granodiorite [13]) were generated in late subduction to continental collision setting [23] or transitional tectonic setting from syn-to- post collision [75]. Recently, Deng et al. [18] proposed that the Lincang granites recorded three major episodes of subduction (261-252 Ma), syn-collision (250-237 Ma), and post-collision (235-203 Ma), respectively. Xin et al. [17] have suggested that the volcanic rocks of the Weixi area in north Lancangjiang were generated in a syn-subduction setting.

The Zengudi and Tuobake granite porphyries are located in the west margin of the Jiangda-Weixi arc volcanic belt (see Figure 1b), which represents an important part of the east Tethys. As mentioned above, the granite porphyries of the Zengudi and Tuobake are medium- to high-potassium, calc-alkaline, and S-type granite porphyries with intrusive ages of ca. 254-247 Ma and $246 \mathrm{Ma}$, respectively. We suggest that the Early Triassic granite porphyries of this study were probably formed in a volcanic arc setting for three reasons: (1) these rocks are characterized by high $\mathrm{Al}_{2} \mathrm{O}_{3}$ contents and LILE/HFSE and $\mathrm{La}_{\mathrm{N}} / \mathrm{Yb}_{\mathrm{N}}$ ratios, low $\mathrm{Ni}$ and $\mathrm{Cr}$ contents and $(\mathrm{Nb} / \mathrm{La})_{\mathrm{N}}$ ratios, and conspicuous negative $\mathrm{Nb}, \mathrm{Ta}, \mathrm{Ti}$, and $\mathrm{P}$ anomalies. All these features indicate a typical arc magma affinity for the studied granites (Figure 13a,b). (2) The Zengudi and Tuobake granite porphyries have similar intrusive age (254-246 Ma) to that 251-244 Ma of the bimodal volcanic rocks from the Yezhi area and other parts of the Jiangda-Weixi volcanic arc which formed under a volcanic arc setting [14-18].(3) Previous studies proposed that the Late Permian to late Early Triassic and Middle to Late Triassic rocks were separately formed within volcanic arc setting and syn-collision to post-collision tectonic settings $[14,15,18,23]$. The granite porphyries of this study were synchronously generated with the Lincang S-type arc granites from the southern Lancangjiang [18].
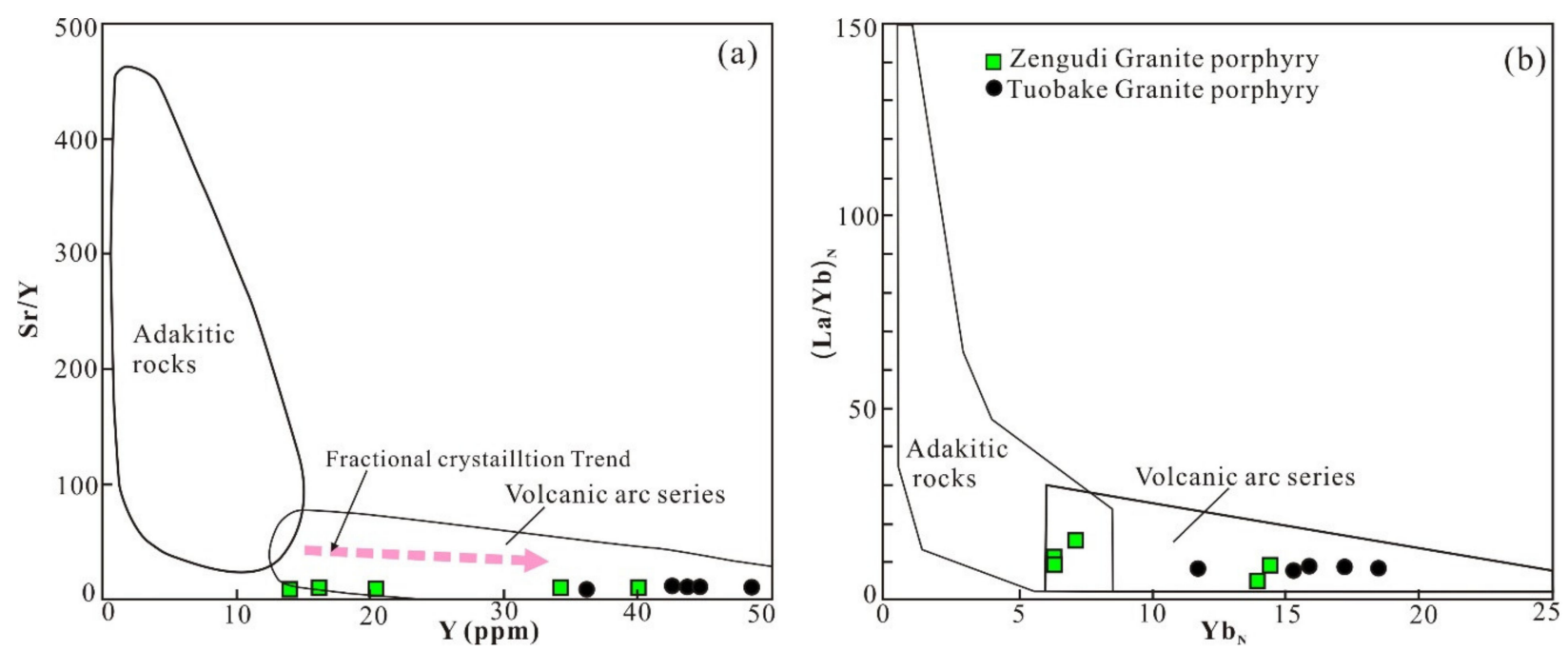

Figure 13. Petrogenesis classification diagrams of the Zengudi and Tuobake granite porphyries. (a) Sr/Y versus Y and (b) $(\mathrm{La} / \mathrm{Yb})_{\mathrm{N}}$ versus $\mathrm{Yb}_{\mathrm{N}}[86]$.

Moreover, trace elements can further provide useful information to constrain the tectonic setting of granitoids [87]. In the discrimination diagrams of $\mathrm{Rb}$ versus $\mathrm{Y}+\mathrm{Nb}$ and $\mathrm{Nb}$ versus $\mathrm{Y}$ (Figure 14a,b), most of the Zengudi and Tuobake granite porphyry samples plot in the volcanic arc field. Comparable results have been obtained using the Rb-Hf-Ta tectonic discrimination diagrams (Figure 14c,d), which confirm that the studied granite porphyries were generated in a volcanic arc setting. Therefore, a volcanic arc setting is the most plausible explanation for the generation of Late Permian to Early Triassic magmatism in the Yezhi area in the northern Lancangjiang zone. 

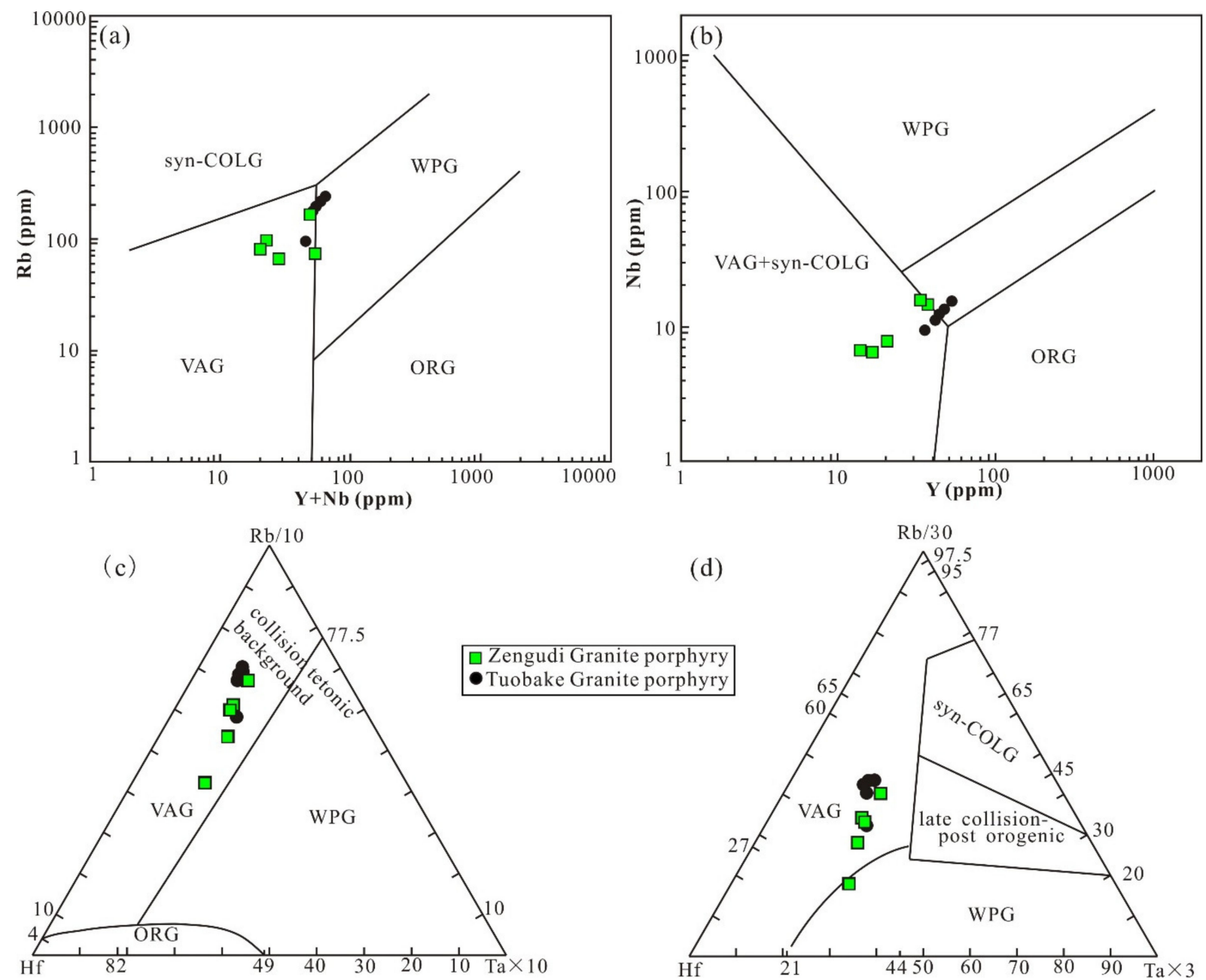

(d)

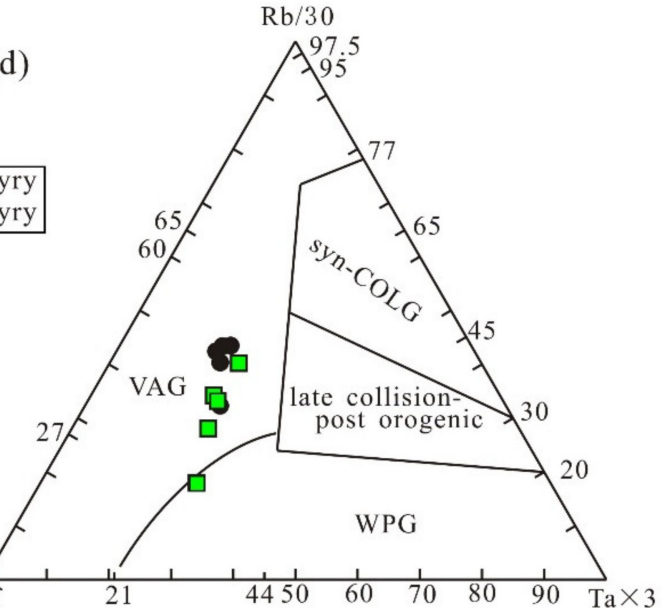

Figure 14. Tectonic classification diagrams of the Zengudi and Tuobake granite porphyries. (a) $\mathrm{Rb}$ versus $\mathrm{Y}+\mathrm{Nb}$ ([88], (b) $\mathrm{Nb}$ versus $\mathrm{Y}$ [87], (c) Hf-Rb/10-Ta $\times 10$ [89], and (d) Hf-Rb/30-Ta $\times 3$ [89]. VAG = volcanic arc granites; ORG = ocean ridge granites; WPG = within-plate granites; syn-COLG = syn-collisional granites; post-COLG = post-collisional granites.

Previous research has suggested that the breakup of the Paleo-Tethys Ocean in the Lancangjiang area started in the Late Devonian and the closure of this ocean occurred in the Late Triassic $[17,30,31]$. In the eastern margin of the Qamdo-Simao block (Figure 1b), the age of the Jiangda-Weixi volcanism ranges from 258 to 229 Ma $[16,19,20,85]$. The emplacement ages of the volcanic rocks from the western margin of the Simao Basin range from 238 to $229 \mathrm{Ma}$. These rocks were considered to be formed by southwestward to northeastward subduction of the Qamdo-Changning-Menglian Paleo-Tethys oceanic basin [34].

In this study, the Zengudi and Tuobake granite porphyries have emplacement ages of Late Permian (254 Ma) to Early Triassic (246 Ma), which are comparable to the SHRIMP zircon $\mathrm{U}-\mathrm{Pb}$ age (249 Ma) obtained for the adjacent Baimaxueshan pluton $[19,20]$ and bimodal volcanic rocks $[16,17]$. Hence, our new geochronological data confirm that the magma emplacement in this area corresponds to the early phase of the Indosinian Orogeny. Therefore, we suggest that the S-type magmatism was synchronous to the tectonic extrusion in the Jiangda-Weixi volcanic arc $[16,85]$, and the shortened and thickened crust in the continental arc was formed by compression. During the Late Permian to Late Triassic, the Lancangiiang and Ailaoshan Oceans subducted beneath the Baoshan and Simao blocks, respectively, while the Songma Ocean experienced a southward subduction beneath the Indochina block. 
Overall, the Zengudi and Tuobake granite porphyries (254-246 Ma), the Baimaxueshan granite (249 Ma) and the Lincang granite (250 Ma) from the northern Sanjang area were synchronously emplaced within the Jiangda-Weixi volcanic arc $[17,39]$. Subduction eastward of the Paleo-Tethys Ocean beneath the Indochina block began before $254 \mathrm{Ma}$ (Figure 15a). From 254 to $246 \mathrm{Ma}$, the continuous subduction of the Paleo-Tethys Ocean led to a strong extrusion and slab break-off of oceanic lithosphere [90] (Figure 15b). During this late stage, partial melting of the thickened crust led to the formation of the large-scale S-type magmas, resulting in the synchronous emplacement of the Zengudi and Tuobake granite porphyries and the eruption of a large volume of volcanic rocks within the Jiangda-Weixi volcanic arc.
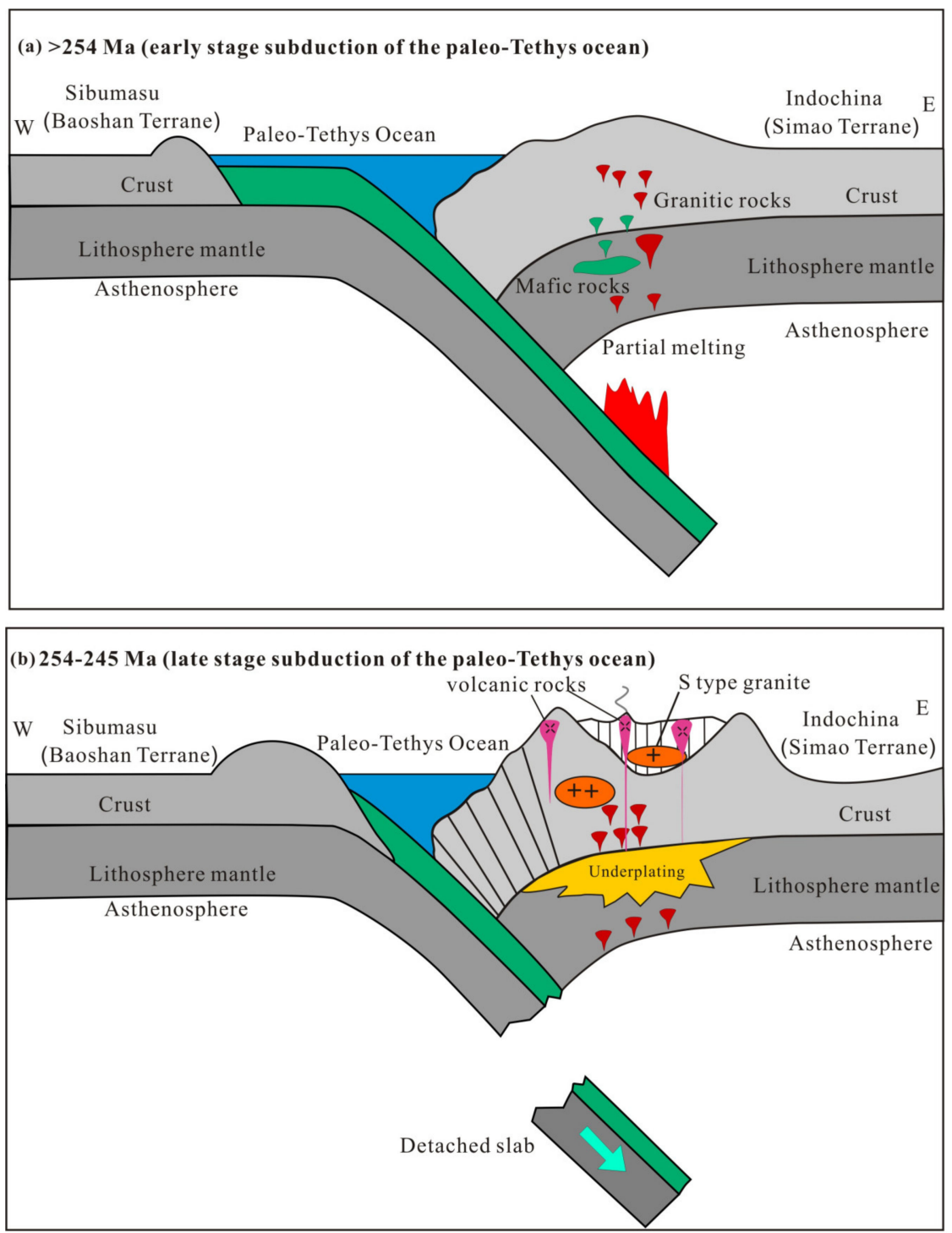

Figure 15. A schematic tectonic cartoon shows the Paleotethys evolution of the northern Lancangjiang tectonic zone. (a) $>245 \mathrm{Ma}$ (early stage subduction of the paleo-Tethys ocean) (b) 254-245 Ma (late stage subduction of the paleo-Tethys ocean).

\section{Conclusions}

1. The U-Pb dating of the Zengudi and Tuobake granite porphyries from the Sanjiang area reveal that these granites were emplaced between 254 and $246 \mathrm{Ma}$, indicating that their emplacement occurred at the closure of the Paleo-Tethys Ocean during Late Permian to Early Triassic. 
2. Whole-rock geochemistry and zircon $\mathrm{Hf}$ isotopic compositions suggest that the granite porphyries are typical volcanic arc rocks formed in the continental arc setting after the subduction of oceanic crust. These rocks were originated from plagioclase-rich and clay-poor clastic source magmas which have likely undergone intensive fractional crystallization.

3. The crust of the Jiangda-Weixi volcanic arc belt was thickened by eastward subduction of the Baoshan block during the closure of the Paleo-Tethys, which resulted in partial melting of the thickened crust.

Author Contributions: Conceptualization, M.-J.T. and H.L.; investigation, M.-J.T.; performed the experiments, M.-J.T.; Analyzed the data, M.-J.T. and H.L.; writing—original draft, M.-J.T., H.L., L.S.T., and Z.X. All authors have read and agreed to the published version of the manuscript.

Funding: This research was supported by funds from the National Natural Science Foundation of China (Grant No. 41774149).

Institutional Review Board Statement: Not Applicable.

Informed Consent Statement: Not Applicable.

Data Availability Statement: All data generated or analysed during this study are included in this published article.

Acknowledgments: The authors would like to thank Duan, J.R (Central South University) for fruitful discussion during the initial preparation of the manuscript. We are grateful to three anonymous reviewers and the Editor-in-Chief for their valuable comments and suggestions that improve the quality of this paper.

Conflicts of Interest: The authors declare no conflict of interest.

\section{References}

1. Wu, J.H.; Li, H.; Xi, X.S.; Kong, H.; Wu, Q.H.; Peng, N.L.; Wu, X.M.; Cao, J.Y.; Gabo-Ratio, J.A.S. Geochemistry and geochronology of the mafic dikes in the Taipusi area, northern margin of North China Craton: Implications for Silurian tectonic evolution of the Central Asian Orogen. J. Earth Syst. Sci. 2017, 64, 126. [CrossRef]

2. Kong, H.; Li, H.; Wu, Q.H.; Xi, X.S.; Dick, J.M.; Gabo-Ratio, J.A.S. Co-development of Jurassic I-type and A-type granites in southern Hunan, South China: Dual control by plate subduction and intraplate mantle upwelling. Chem. Erde Geoch. 2018, 78, 500-520. [CrossRef]

3. Zheng, Y.F.; Xu, Z.; Chen, L.; Dai, L.Q.; Zhao, Z.F. Chemical geodynamics of mafic magmatism above subduction zones. J. Asian Earth Sci. 2020, 194, 104185. [CrossRef]

4. Li, H.; Xi, X.S.; Wu, C.M.; Zhang, D.B. Geochemistry and genesis of andesite from Zhaokalong Fe-Cu polymetallic deposit, Yushu, Qinghai. J. Cent. South. Univ. 2012, 43, 3524-3534, (in Chinese with English abstract).

5. Li, H.; Xi, X.S.; Sun, H.S.; Kong, H.; Wu, Q.H.; Wu, C.M.; Gabo-Ratio, J.A.S. Geochemistry of the Batang Group in the Zhaokalong area, Yushu, Qinghai: Implications for the Late Triassic tectonism in the northern Sanjiang region, China. Acta Geol. Sin. Engl. 2016, 90, 704-721.

6. Deng, J.; Wang, Q.F.; Li, G.J.; Li, C.S.; Wang, C.M. Tethys tectonic evolution and its bearing on the distribution of important mineral deposits in the Sanjiang region, SW China. Gondwana Res. 2014, 26, 419-437. [CrossRef]

7. Sun, H.S.; Li, H.; Danišík, M.; Xia, Q.L.; Jiang, C.L.; Wu, P.; Yang, H.; Fan, Q.R.; Zhu, D.S. U-Pb and Re-Os geochronology and geochemistry of the Donggebi Mo deposit, Eastern Tianshan, NW China: Insights into mineralization and tectonic setting. Ore Geol. Rev. 2017, 86, 584-599. [CrossRef]

8. Zhai, M.G.; Zhang, Q.; Chen, G.N.; Wang, R.C. Adventure on the research of continental evolution and related granite geochemistry. Chinese Sci. Bull. 2016, 61, 1414-1420.

9. Cao, J.; Wu, Q.; Yang, X.; Kong, H.; Li, H.X.; Liu, B. Geochronology and genesis of the Xitian W-Sn polymetallic deposit in eastern Hunan Province, South China: Evidence from zircon U-Pb and muscovite Ar-Ar dating, petrochemistry, and wolframite $\mathrm{Sr}-\mathrm{Nd}-\mathrm{Pb}$ isotopes. Minerals 2018, 8, 111. [CrossRef]

10. Li, H.; Palinkaš, L.A.; Watanabe, K.; Xi, X.S. Petrogenesis of Jurassic A-type granites associated with Cu-Mo and W-Sn deposits in the central Nanling region, South China: Relation to mantle upwelling and intra-continental extension. Ore Geol. Rev. 2018, 92, 449-462. [CrossRef]

11. Wang, T.; Huang, C.; Du, G.; Liu, Y.; Xie, J.; Li, H. Geochronology, geochemistry and zircon Hf isotopes of the early Mesoproterozoic Yaopengzi dolerite in SW Yangtze block (Sichuan, SW China): Implications for the Columbia supercontinent breakup. Geosci. J. 2019, 23, 557-573. [CrossRef] 
12. Jian, P.; Liu, D.Y.; Kroner, A.; Zhang, Q.; Wang, Y.; Sun, X.; Zhang, W. Devonian to Permian plate tectonic cycle of the Paleo-Tethys Orogen in southwest China (I): Geochemistry of ophiolites, arc/back-arc assemblages and within-plate igneous rocks. Lithos 2009, 113, 748-766. [CrossRef]

13. Hennig, D.; Lehmann, B.; Frei, D.; Belyatsky, B.; Zhao, X.F.; Cabral, A.R.; Zeng, P.S.; Zhou, M.F.; Schmidt, K. Early Permian seafloor to continental arc magmatism in the eastern Paleo-Tethys: $\mathrm{U}-\mathrm{Pb}$ age and $\mathrm{Nd}-\mathrm{Sr}$ isotope data from the southern Lancangjiang zone, Yunnan, China. Lithos 2009, 113, 408-422. [CrossRef]

14. Peng, T.P.; Wang, Y.J.; Zhao, G.C.; Fan, W.M.; Peng, B.X. Arc-like volcanic rocks from the southern Lancangjiang zone, SW China: Geochronological and geochemical constraints on their petrogenesis and tectonic implications. Lithos 2008, 102, 358-373. [CrossRef]

15. Peng, T.P.; Wilde, S.A.; Wang, Y.J.; Fan, W.M.; Peng, B.X. Mid-Triassic felsic igneous rocks fromthe southern Lancangjiang Zone, SW China: Petrogenesis and implications for the evolution of Paleotethys. Lithos 2013, 168-169, 15-32. [CrossRef]

16. Wang, B.D.; Wang, L.Q.; Chen, J.L.; Yin, F.G.; Wang, D.B.; Zhang, W.P.; Chen, L.K.; Liu, H. Triassic three-stage collision in the Paleo-Tethys: Constraints from magmatism in the Jiangda-Deqen-Weixi continental margin arc, SW China. Gondwana Res. 2014, 26, 475-491. [CrossRef]

17. Xin, D.; Yang, T.N.; Liang, M.J.; Xue, C.D.; Tang, J. Syn-subduction crustal shortening produced a magmatic flare-up in middle Sanjiang orogenic belt, southeastern Tibet Plateau: Evidence from geochronology, geochemistry, and structural geology. Gondwana Res. 2018, 62, 93-111. [CrossRef]

18. Deng, J.; Wang, C.M.; Zi, J.W.; Xia, R.; Li, Q. Constraining subduction-collision processes of the Paleo-Tethys along the ChangningMenglian Suture: New zircon U-Pb ages and Sr-Nd-Pb-Hf-O isotopes of the Lincang Batholith. Gondwana Res. 2018, 62, 75-92. [CrossRef]

19. Zi, J.W.; Cawood, P.A.; Fan, W.M.; Wang, Y.J.; Tohver, E.; McCuaig, T.C. Generation of Early Indosinian enriched mantle-derived granitoid pluton in the Sanjiang Orogen (SW China) in response to closure of the Paleo-Tethys. Lithos 2012, 140, 166-182. [CrossRef]

20. Zi, J.W.; Cawood, P.A.; Fan, W.M.; Wang, Y.J.; Tohver, E.; McCuaig, T.C.; Peng, T.P. Triassic collision in the Paleo-Tethys Ocean constrained by volcanic activity in SW China. Lithos 2012, 144, 145-160. [CrossRef]

21. Yang, T.N.; Zhang, H.R.; Fan, J.W.; Liang, M.J. Two-phase subduction and subsequent collision defines the Paleo-tethyan tectonics of the southeastern Tibetan Plateau: Evidence from zircon U-Pb dating, geochemistry, and structural geology of the Sanjiang orogenic belt, southwest China. Geol. Soc. Am. 2014, 126, 1654-1682. [CrossRef]

22. He, W.Y.; Yang, L.Q.; Lu, Y.; Heon, H.; Xie, S.; Gao, X. Zircon U-Pb dating, geochemistry and Sr-Nd-Hf-O isotopes for the Baimaxueshan granodiorites and mafic microgranulars enclaves in the Sanjiang Orogen: Evidence for westward subduction of Paleo-Tethys. Gondwana Res. 2018, 62, 112-126. [CrossRef]

23. Dong, G.C.; Mo, X.X.; Zhao, Z.D.; Zhu, D.C.; Goodman, R.; Kong, H.L.; Wang, S. Zircon U-Pb dating and the petrological and geochemical constraints on Lincang granite in Western Yunnan, China: Implications for the closure of the Paleo-Tethys Ocean. J. Asian Earth Sci. 2013, 62, 282-294. [CrossRef]

24. Metcalfe, I. Palaeozoic and Mesozoic tectonic evolution and palaeogeography of East Asian crustal fragments: The Korean Peninsula in context. Gondwana Res. 2006, 9, 24-46. [CrossRef]

25. Li, W.C.; Yin, G.H.; Yu, H.J.; Lu, Y.X.; Liu, X.L. The porphyry metallogenesis of Geza volcanic magmatic arc in NW Yunnan. Acta Petrol. Sin. 2010, 27, 2541-2552. (In Chinese)

26. Feng, Q.L. Stratigraphy of volcanic rocks in the Changning-Menlian Belt in southwestern Yunnan, China. J. Asian Earth Sci. 2002, 20, 657-664. [CrossRef]

27. Yan, Q.R.; Wang, Z.Q.; Liu, S.W.; Li, Q.G.; Zhang, H.Y.; Wang, T.; Liu, D.Y. Opening of the Tethys in southwest China and its significance to the breakup of East Gondwana and in the late Paleozoic: Evidence from SHRIMP U-Pb zircon analyses for the Garze ophiolite block. Chin. Sci. Bull. 2005, 50, 256-264. [CrossRef]

28. Zhai, Q.G.; Jahn, B.M.; Su, L.; Richard, E.E.; Wang, K.L.; Zhang, R.Y.; Wang, J.; Tang, S.H. SHRIMP zircon U-Pb geochronology, geochemistry and $\mathrm{Sr}-\mathrm{Nd}-\mathrm{Hf}$ isotopic compositions of a mafic dyke swarm in the Qiangtang terrane, northern Tibet and geodynamic implications. Lithos 2013, 174, 28-43. [CrossRef]

29. Zhai, Q.G.; Jahn, B.M.; Su, L.; Wang, J.; Mo, X.X.; Lee, H.Y. Triassic arc magmatism in the Qiangtang area, northern Tibet: Zircon $\mathrm{U}-\mathrm{Pb}$ ages, geochemical and Sr-Nd-Hf isotopic characteristics, and tectonic implications. J. Asian Earth Sci. 2013, 63, 162-178. [CrossRef]

30. Jian, P.; Liu, D.Y.; Sun, X.M. SHRIMP dating of the Permo-Carboniferous Jinshajiang ophiolite, southwest China: Geochronological constraints for the evolution of Paleo-Tethys. J. Asian Earth Sci. 2008, 32, 371-384. [CrossRef]

31. Jian, P.; Liu, D.Y.; Kroner, A.; Zhang, Q.; Wang, Y.; Sun, X.; Zhang, W. Devonian to Permian plate tectonic cycle of the Paleo-Tethys Orogen in southwest China (II): Insights from zircon ages of ophiolites, arc/back-arc assemblages and within-plate igneous rocks and generation of the Emeishan CFB province. Lithos 2009, 113, 767-784. [CrossRef]

32. Wang, X.F.; Metcalfeb, I.; Jian, P.; He, L.Q.; Wang, C.S. The Jinshajiang-Ailaoshan Suture Zone, China: Tectonostratigraphy, age and evolution. J. Asian Earth Sci. 2000, 18, 675-690. [CrossRef]

33. Yang, T.N.; Zhang, H.R.; Song, Y.C.; Tian, S.H. Permo-Triassic arc magmatism in central Tibet: Evidence from zircon U-Pb geochronology, Hf isotopes, rare earth elements, and bulk geochemistry. Chem. Geol. 2011, 28, 270-282. [CrossRef] 
34. Rossignol, C.; Bourquin, S.; Hallot, E.; Poujol, M.; Dabard, M.-P.; Martini, R.; Villeneuve, M.; Cornée, J.-J.; Brayard, A.; Roger, F. The Indosinian orogeny: A perspective from sedimentary archives of north vietnam. J. Asian Earth Sci. 2018, 158, 352-380. [CrossRef]

35. Villeneuve, M.; Rossignol, C.; Martini, R.; Cornée, J.-J.; Bourquin, S. New insights into the Triassic sedimentary environment of the eastern parts of the Song Da and Sam Nua basins alongside the Indosinian Song Ma suture, Northern Vietnam. J. Asian Earth Sci. 2020, 187, 104067. [CrossRef]

36. Fan, W.M.; Wang, Y.J.; Zhang, Y.H.; Zhang, Y.Z.; Jourdan, F.; Zi, J.W.; Liu, H.C. Paleotethyan subduction process revealed from Triassic blueschists in the Lancang tectonic belt of Southwest China. Tectonophysics 2015, 662, 95-108. [CrossRef]

37. Xiong, Y.Q.; Shao, Y.J.; Mao, J.W.; Wu, S.C.; Zhou, H.D.; Zheng, M.H. The polymetallic magmatic-hydrothermal Xiangdong and Dalong systems in the $\mathrm{W}-\mathrm{Sn}-\mathrm{Cu}-\mathrm{Pb}-\mathrm{Zn}-\mathrm{Ag}$ Dengfuxian ore field, SE China: Constraints from geology, fluid inclusions, $\mathrm{H}-\mathrm{O}-\mathrm{S}-\mathrm{Pb}$ isotopes, and sphalerite $\mathrm{Rb}-\mathrm{Sr}$ geochronology. Miner. Deposita 2019, 54, 1101-1124. [CrossRef]

38. Lin, J.; Liu, Y.S.; Yang, Y.H.; Hu, Z.C. Calibration and correction of LA-ICP-MS and LA-MC-ICP-MS analyses for element contents and isotopic ratios. Solid Earth Sci. 2016, 1, 5-27. [CrossRef]

39. Xiong, Y.Q.; Jiang, S.Y.; Wen, C.H.; Yu, H.Y. Granite-pegmatite connection and mineralization age of the giant Renli Ta-Nb deposit in South China: Constraints from U-Th-Pb geochronology of coltan, monazite, and zircon. Lithos 2020, 358-359, 105422. [CrossRef]

40. Liu, Y.S.; Hu, Z.C.; Gao, S.; Xu, J.; Gao, C.G.; Chen, H.H. In Situ Analysis of Major and Trace Elements of Anhydrous Minerals by LA-ICP-MS without Applying an Internal Standard. Chem. Geol. 2008, 257, 34-43. [CrossRef]

41. Liu, Y.S.; Gao, S.; Hu, Z.C.; Gao, C.G.; Zong, K.Q.; Wang, D.B. Continental and Oceanic Crust Recycling-Induced Melt-Peridotite Interactions in the Trans-North China Orogen: U-Pb Dating, Hf Isotopes and Trace Elements in Zircons from Mantle Xenoliths. J. Petrol. 2009, 51, 537-571. [CrossRef]

42. Hu, Z.C.; Gao, S.; Liu, W.G.; Zhang, W.; Tong, X.R.; Lin, L.; Zong, K.Q.; Li, M.; Chen, H.H.; Zhong, L.; et al. Improved situ Hf isotope ratio analysis of zircon using newly designed $X$ skimmer cone and jet sample cone in combination with the addition of nitrogen by Laser ablation multiple collector ICP-MS. J. Anal. Spectrom. 2012, 27, 1391-1399. [CrossRef]

43. Fisher, C.M. Guidelines for reporting zircon Hf isotopic data by LA-MC-ICPMS and potential pitfalls in the interpretation of these data. Chem. Geol. 2014, 363, 125-133. [CrossRef]

44. Blichert-Toft, J.; Albarede, F. The Lu-Hf isotope geochemistry of chondrites and the evolution of the mantle-crust system. Earth Planet. Sci. Lett. 1997, 148, 243-258. [CrossRef]

45. Middlemost, E.A.K. Naming materials in the magma/igneous rock system. Earth Sci. Rev. 1994, 37, 215-224. [CrossRef]

46. Peccerillo, R.; Taylor, S.R. Geochemistry of Eocene calc-alkaline volcanic rocks from the Kastamonu area, Northern Turkey. Contrib. Mineral. Petr. 1976, 58, 63-81. [CrossRef]

47. Maniar, P.D.; Piccoli, P.M. Tectonic discrimination of granitoids. Geol. Soc. Am. Bull. 1989, 101, 635-643. [CrossRef]

48. Rollinson, H. Using Geochemical Data: Evaluation, Presentation, Interpretation; Longman Scientific \& Technical: London, UK, 1993; pp. $1-352$.

49. Wright, J.B. A simple alkalinity ratio and its application to questions of non-orogenic granite genesis. Geol. Mag. 1969, 106, 370-384. [CrossRef]

50. Sun, X.S.; McDonough, W.F. Chemical and Isotopic Systematics of Oceanic Basalts: Implications for Mantle Composition and Processes. Geological Society, London. Spec. Publ. 1989, 42, 313-345. [CrossRef]

51. Corfu, F.; Hanchar, J.M.; Hoskin, P.W.O.; Kinny, P. Atlas of zircon textures. Rev. Mineral. Geochem. 2003, 53, 469-500. [CrossRef]

52. Xiong, Y.Q.; Shao, Y.J.; Cheng, Y.; Jiang, S.Y. Discrete Jurassic and Cretaceous Mineralization Events at the Xiangdong W(-Sn) Deposit, Nanling Range, South China. Econ. Geol. 2020, 115, 385-413. [CrossRef]

53. Li, H.; Sun, H.S.; Evans, N.J.; Li, J.W.; Wu, J.H.; Jiang, W.C.; Halassane, N. Geochemistry and geochronology of zircons from granite-hosted gold mineralization in the Jiaodong Peninsula, North China: Implications for ore genesis. Ore Geol. Rev. 2019, 115, 103188. [CrossRef]

54. Li, H.; Danišík, M.; Zhou, Z.K.; Jiang, W.C.; Wu, J.H. Integrated U-Pb, Lu-Hf and (U-Th)/He analysis of zircon from the Banxi Sb deposit and its implications for the low-temperature mineralization in South China. Geosci. Front. 2020, 11, 1323-1335. [CrossRef]

55. Belousova, E.A.; Griffin, W.; Suzanne, Y.; O'Reilly, F.N. Igneous zircon: Trace element composition as an indicator of source rock type. Contrib. Mineral. Petr. 2002, 143, 602-622. [CrossRef]

56. Wu, F.Y.; Li, X.H.; Zheng, Y.F.; Gao, S. Lu-Hf isotopic systematics and their applications in petrology. Acta Petrol. Sin. 2007, 23, 185-220, (In Chinese with English abstract).

57. Chappell, B.W.; White, A.J.R. Two contrasting granite types. Pac. Geol. 1974, 8, 173-174.

58. Loiselle, M.C.; Wones, D.R. Characteristics and origin of anorogenic granites. Geol. Soc. Am. Abstr. Programs 1979, $11,468$.

59. Chappell, B.W.; White, A.J.R. I- and S-type granites in the Lachlan Fold Belt. Trans. R. Soc. Edinb. Earth Sci. 1992, 83, 1-26.

60. Bonin, B. A-type granites and related rocks: Evolution of a concept, problems and prospects. Lithos 2007, 97, 1-29. [CrossRef]

61. Whalen, J.B.; Currie, K.L.; Chappell, B.W. A-type granites: Geochemical characteristics, discrimination and petrogenesis. Contrib. Mineral. Petr. 1987, 95, 407-419. [CrossRef]

62. Li, H.; Watanabe, K.; Yonezu, K. Geochemistry of A-type granites in the Huangshaping polymetallic deposit (South Hunan, China): Implications for granite evolution and associated mineralization. J. Asian Earth Sci. 2014, 88, 149-167. [CrossRef] 
63. Girei, M.B.; Li, H.; Algeo, T.J.; Bonin, B.; Ogunleye, P.O.; Bute, S.I.; Ahmed, H.A. Petrogenesis of A-type granites associated with $\mathrm{Sn}-\mathrm{Nb}-\mathrm{Zn}$ mineralization in Ririwai complex, north-Central Nigeria: Constraints from whole-rock Sm-Nd and zircon Lu-Hf isotope systematics. Lithos 2019, 340-341, 49-70. [CrossRef]

64. Chappell, B.W. Aluminium saturation in I- and S-type granites and the characterization of fractionated haplogranites. Lithos 1999, 46, 535-551. [CrossRef]

65. Pefford, N.; Atherton, M. Na-rich Partial Melts from Newly Underplated Basaltic Crust: The Cordillera Blanca Batholith, Peru. J. Petrol. 1996, 37, 1491-1521. [CrossRef]

66. Sylvester, P.J. Post-Collisional Strongly Peraluminous Granites. Lithos 1998, 45, 29-44. [CrossRef]

67. Li, H.; Wu, J.H.; Evans, N.J.; Jiang, W.C.; Zhou, Z.K. Zircon geochronology and geochemistry of the Xianghualing A-type granitic rocks: Insights into multi-stage Sn-polymetallic mineralization in South China. Lithos 2018, 312-313, 1-20. [CrossRef]

68. Clemens, J.D. S-type granitic magmas-petrogenetic issues, models and evidence. Earth Sci. Rev. 2003, 61, 1-18. [CrossRef]

69. Douce, A.E.P.; Beard, J.S. Dehydration-melting of Biotite Gneiss and Quartz Amphibolite from 3 to 15 kbar. J. Petrol. 1995, 36, 707-738. [CrossRef]

70. Springer, W.; Seek, H.A. Partial fusion of basic granulites at 5 to 15 kbar: Implications for the origin of TTG magmas. Contrib. Min. Petr. 1997, 127, 30-45. [CrossRef]

71. Holtz, F.; Johannes, W.; Tamic, N. Maximum and minimum water contents of granitic melts generated in the crust: A reevaluation and implications. Lithos 2001, 56, 1-14. [CrossRef]

72. Douce, A.E.P.; Johnston, A.D. Phase equilibria and melt productivity in the pelitic system: Implications for the origin of peraluminousgranitoids and aluminous granulites. Contrib. Min. Petr. 1991, 107, 202-218. [CrossRef]

73. Cisse, M.; Lu, X.; Algeo, T.J.; Cao, X.; Li, H.; Wei, M.; Yuan, Q.; Chen, M. Geochronology and geochemical characteristics of the Dongping ore-bearing granite, North China: Sources and implications for its tectonic setting. Ore Geol. Rev. 2017, 89, 1091-1106. [CrossRef]

74. $\mathrm{Xu}$, J.F.; Castillo, P.R. Geochemical and $\mathrm{Nd}-\mathrm{Pb}$ isotopic characteristics of the Tethyan asthenosphere: Implications for the origin of the Indian Ocean mantle domain. Tectonophysics 2004, 393, 9-27. [CrossRef]

75. Ma, L.Y.; Wang, Y.J.; Fan, W.M.; Geng, H.Y.; Cai, Y.F.; Zhong, H.; Liu, H.C.; Xing, X.W. Petrogenesis of the early Eocene I-type granites in West Yingiang (SW Yunnan) and its implication for the eastern extension of the Gangdese batholiths. Gondwana Res. 2014, 25, 401-419. [CrossRef]

76. Healy, B.; Collins, W.J.; Richards, S.W. A hybrid origin for Lachlan S-type granites: The Murrumbidgee batholith example. Lithos 2004, 78, 197-216. [CrossRef]

77. Hao, T.P. Sm-Nd isotopic ages of Proterozoic metamorphic rocks from the middle sector of the Jinsha River. Geol. Rev. 1993, 39, 52-56. (In Chinese)

78. Gao, S.; Ling, W.L.; Qiu, Y.M.; Lian, Z.; Hartmann, G.; Klaus, S. Contrasting geochemical and Sm-Nd isotopic compositions of Archean metasediments from the Kongling high-grade terrain of the Yangtze craton: Evidence for cratonic evolution and redistribution of REE during crustal anatexis. Geochim. Cosmochim. Acta 1999, 63, 2071-2088. [CrossRef]

79. Taylor, S.R.; McLennan, S.M. The Continental Crust: Its Composition and Evolution; Blackwell, Oxofrd Press: Oxofrd, UK, 1985; pp. $1-312$.

80. Green, T.H. Significance of $\mathrm{Nb} / \mathrm{Ta}$ as an indicator of geochemical processe in the crust-mantle system. Chem. Geol. 1995, 120, 347-359. [CrossRef]

81. Griffin, W.L.; Pearson, N.J.; Belousova, E.; Jackson, S.E.; O’Reilly, S.Y.; Van Achterberg, E.; Shee, S.R. The Hf isotope composition of cratonic mantle: LAM-MC-ICP MS analysis of zircon megacrysts in kimberlites. Geochim. Cosmochim. Acta 2000, 64, 133-147. [CrossRef]

82. Guo, Z.F.; Hertogen, J.; Liu, J.Q. Potassic magmatism in Western Sichuan and Yunnan Provinces, SE Tibet, China: Petrological and geochemical constraints on petrogenesis. J. Petrol. 2005, 46, 33-78. [CrossRef]

83. Hu, Z.L.; Yang, X.Y.; Duan, L.Y.; Sun, W.D. Geochronological and geochemical constraints on genesis of the adakitic rocks in Outang, South Tan-Lu Fault Belt (Northeastern Yangtze Block). Tectonophysics 2014b, 626, 86-104. [CrossRef]

84. Zi, J.W.; Cawood, P.A.; Fan, W.M.; Tohver, E.; Wang, Y.J.; McCuaig, T.C.; Peng, T.P. LatePermian-Triassic magmatic evolution in the Jinshajiang orogenic belt, SW China and implication for orogenic processes following closure of the Paleo-Tethys. Am. J. Sci. 2013, 313, 81-112. [CrossRef]

85. Metcalfe, I. Permian tectonic framework and paleogeography of SE Asia. J. Asian Earth Sci. 2002, 20, 551-566. [CrossRef]

86. Defant, M.J.; Drummond, M.S. Derivation of Some Modern Arc Magmas by Melting of Young Subducted Lithosphere. Nature 1990, 347, 662-665. [CrossRef]

87. Pearce, J.A.; Harris, N.B.W.; Tindle, A.G. Trace Element Discrimination Diagrams for the Tectonic Interpretation of Granitic Rocks. J. Petrol. 1984, 25, 956-983. [CrossRef]

88. Pearce, J. Sources and Settings of Granitic Rocks. Episodes 1996, 19, 120-125. [CrossRef]

89. Harries, N.B.W.; Pearce, J.A.; Tindle, A.G. Geochemical characterristics of collision-zone magmatism. Spec. Publ. 1986, 19 , 67-81. [CrossRef]

90. Zhang, W.; Lentz, D.R.; Thorne, K.G.; Massawe, R.J.R. Late Silurian-Early Devonian slab break-off beneath the Canadian Appalachians: Insights from the Nashwaak Granite, west-central New Brunswick, Canada. Lithos 2020, 358-359, 105393. [CrossRef] 